\title{
The dark matter halo shape of edge-on disk galaxies
}

\section{Modelling the HI observations: methods}

\author{
J. C. O’Brien ${ }^{1}$, K. C. Freeman ${ }^{1}$, and P. C. van der Kruit ${ }^{2}$ \\ 1 Research School of Astronomy and Astrophysics, Australian National University, Mount Stromlo Observatory, Cotter Road, \\ ACT 2611, Australia \\ e-mail: jesscobrien@gmail.com; kcf@mso.anu.edu.au \\ 2 Kapteyn Astronomical Institute, University of Groningen, PO Box 800, 9700 AV Groningen, The Netherlands \\ e-mail: vdkruit@astro.rug.nl
}

Received 25 May 2009 / Accepted 9 March 2010

\section{ABSTRACT}

\begin{abstract}
This is the second paper of a series in which we attempt to put constraints on the flattening of dark halos in disk galaxies. For this purpose, we observe the $\mathrm{HI}$ in edge-on galaxies, where it is in principle possible to measure the force field in the halo vertically and radially from gas layer flaring and rotation curve decomposition respectively. To calculate the force fields, we need to analyse the observed XV diagrams to accurately measure all three functions that describe the planar kinematics and distribution of a galaxy: the radial HI surface density, the rotation curve and the HI velocity dispersion. In this paper, we discuss the improvements and limitations of the methods previously used to measure these HI properties. We extend the constant velocity dispersion method to include determination of the HI velocity dispersion as a function of galactocentric radius and perform extensive tests on the quality of the fits. We will apply this "radial decomposition XV modelling method" to our HI observations of 8 HI rich, late-type, edge-on galaxies in the third paper of this series.
\end{abstract}

Key words. galaxies: structure - galaxies: kinematics and dynamics - galaxies: halos - galaxies: ISM

\section{Introduction}

In Paper I (O’Brien et al. 2010) in this series we presented HI observations of a sample of 8 edge-on, HI rich, late-type galaxies. The aim of the project has been described there in detail. Briefly, we attempt to put constraints on the flattening of dark halos around disk galaxies by measuring the force field of the halo vertically from the flaring of the HI layer and radially from rotation curve decomposition. For the vertical force field we need to determine in these galaxies both the velocity dispersion of the HI gas (preferably as a function of height from the central plane of the disk) and the thickness of the HI layer, all of this as a function of galactocentric radius. In addition we also need to extract information on the rotation curve of the galaxy and the deprojected HI surface density, as a function of galactocentric radius. In this second paper in the series we will review earlier determinations of these properties and then describe the methods we have developed to analyse our sample.

To meet our goals we need to measure the vertical structure of galaxies out to the lowest surface densities possible and use galaxies with relatively large fractions of their mass in their halos, i.e. high total mass-to-light ratios galaxies. For that purpose we defined a sample of nearby, HI rich, late-type edge-on galaxies. From Paper I we recall that for a vertically isothermal gas sheet with a vertically Gaussian density distribution, the gradient of the total force in the vertical direction can be written as

$\frac{\partial K_{z}}{\partial z}=-\frac{\sigma_{\mathrm{v}, \mathrm{g}}^{2}}{\left(F W H M_{z, \mathrm{~g}} / 2.35\right)^{2}}$,

where $\sigma_{\mathrm{v}, \mathrm{g}}$ is the vertical velocity dispersion of the gas and $F W H M_{z, \mathrm{~g}}$ the gas layer thickness.
The vertical force field comes from the gas itself, plus the dark halo and the stars of the disk. In regions where the halo is a significant contributor to $K_{z}$, the HI flaring will be smaller for the more flattened halos with a smaller axis ratio $q=c / a$ (the ratio of the halo polar axis $c$ to its major axis in the galactic plane $a$ ).

In the present paper we will first present a new method to accurately determine the rotation curve, deprojected HI surface density and HI velocity dispersion at all radii in a edge-on gas disk. The motivation for this measurement method came from the strong dependence in Eq. (2) in Paper I of the derived dark halo flattening $q$ on the measured HI velocity dispersion and vertical HI gas disk flaring. The superposition of velocity profiles from many radii in each sightline through an edge-on HI disk tends to cause an overestimate of the velocity dispersion with most measurement methods, as explained below. Measuring the radial flaring profile requires a model of the galaxy rotation and face-on surface density; this also necessitates high accuracy rotation curve measurement and HI surface density deprojection.

The HI velocity dispersion has not previously been measured systematically in edge-on galaxies. Most measurements of the HI velocity dispersion have been conducted on face-on galaxies, and these measurements were largely conducted $15-20$ years ago with relatively low spatial resolution $F W H M_{\theta}=1-3 \mathrm{kpc}$. In Tables 1 and 2, we show the resolution of each observation used to measure the velocity dispersion of near face-on and near-edge-on galaxies, respectively. The radial structure of the HI velocity dispersion has been measured in only 6 galaxies 5 from near face-on galaxies and one from an edge-on galaxy. The 5 face-on galaxies were observed at $\sim 1 \mathrm{kpc}$ or larger resolution. The uncertain inclination of low resolution face-on HI observations can cause additional broadening, due to projection of 
Table 1. Summary of previous observations of gas velocity dispersion in face-on galaxies.

\begin{tabular}{|c|c|c|c|c|c|c|c|c|c|c|}
\hline \multirow[t]{2}{*}{ Galaxies } & \multirow{2}{*}{$\begin{array}{l}i \\
\circ\end{array}$} & \multirow[t]{2}{*}{ Type } & \multirow{2}{*}{$\begin{array}{c}\text { HI Velocity } \\
\text { dispersion }\end{array}$} & \multicolumn{2}{|c|}{ Resolution } & \multicolumn{3}{|c|}{ Hi radius } & \multirow{2}{*}{$\begin{array}{l}\text { Dist. } \\
\mathrm{Mpc}\end{array}$} & \multirow[t]{2}{*}{ Reference } \\
\hline & & & & $\begin{array}{c}\text { spectral } \\
\mathrm{km} \mathrm{s}^{-1}\end{array}$ & $\begin{array}{c}\text { spatial } \\
\mathrm{kpc}\end{array}$ & $\mathrm{kpc}$ & $R_{25}$ & $\theta_{\text {maj }}$ & & \\
\hline NGC 3938 & $10 \pm 5$ & $\mathrm{ScI}$ & constant $^{h}$ & $8.2^{a}$ & $1.2 \times 1.7$ & 13.1 & 1.7 & 7.5 & 10 & van der Kru \\
\hline NGC 628 & $5 \pm 5^{e}$ & ScI & falling $^{h}$ & $8.2^{a}$ & $2.9 \times 2.9$ & 40.7 & 2.8 & 14.0 & 10 & Shostak \& van der Kruit (1984) \\
\hline NGC 1058 & $8 \pm 2^{f}$ & ScII-III & constant $^{h}$ & $8.2^{a}$ & $2.2 \times 2.2^{c}$ & 18.9 & 4.3 & 8.7 & $10^{c}$ & van der Kruit \& Shostak (1984) \\
\hline NGC 1058 & $8 \pm 2^{f}$ & ScII-III & falling ${ }^{h}$ & $2.58^{a}$ & $2.1 \times 2.0^{d}$ & 14.1 & 3.2 & 6.7 & $10^{d}$ & Dickey et al. (1990) \\
\hline NGC 6946 & 34 & $\mathrm{Scd} / \mathrm{ScI}$ & falling $^{h}$ & $8.25^{a}$ & $0.7 \times 0.8$ & 17.2 & 2.0 & 21.2 & $5.9^{b}$ & Boulanger \& Viallefond (1992) \\
\hline NGC $5474^{g}$ & $21_{-6}^{+4}$ & ScdIV & falling $^{h}$ & $5.2^{a}$ & $1.2 \times 1.1$ & 10.5 & 4.6 & 8.6 & 7 & Rownd et al. (1994) \\
\hline NGC 1058 & $8 \pm 2^{f}$ & ScII-III & falling $^{h}$ & $2.58^{a}$ & $1.5 \times 1.5$ & 21.8 & 5.0 & 15.0 & 10 & Petric \& Rupen (2006) \\
\hline
\end{tabular}

Notes. ${ }^{(a)}$ Hanning smoothed.

(b) Distance shown is mean distance calculated from brightest stars in NGC 6946 group.

(c) To facilitate comparison with the other observations for NGC 1058, we have used the adopted distance of 10 Mpc used by Petric \& Rupen (2006), rather than the van der Kruit \& Shostak (1984) preferred value of 9 Mpc.

(d) To facilitate comparison with the other observations for NGC 1058, we have used the adopted distance of 10 Mpc used by Petric \& Rupen (2006), rather than the Dickey et al. (1990) preferred value of 10.2-14.5 Mpc.

(e) Warps from $\approx 5^{\circ}$ within the optical disk to $i \approx 0^{\circ}$, then back to $i \approx 10^{\circ}$ at the Hi edge (Shostak \& van der Kruit 1984).

(f) Warps from $\approx 8 \pm 2^{\circ}$ within the optical disk to exactly face-on at the Hi edge (van der Kruit \& Shostak 1984).

(g) Classified as peculiar, and known to be interacting with its much larger neighbour M101 which is only 90 kpc distance assuming a distance of 7 Mpc (Rownd et al. 1994).

${ }^{(h)}$ Direct measurement of the velocity dispersion of the broadening of the vertical velocity dispersion from a near face-on disk. Measurements corrected for instrumental broadening and inclination, but not beam-smearing.

Table 2. Summary of previous observations of gas velocity dispersion of highly inclined galaxies.

\begin{tabular}{|c|c|c|c|c|c|c|c|c|c|c|}
\hline \multirow[t]{2}{*}{ Galaxies } & \multirow{2}{*}{$\begin{array}{l}i \\
\circ\end{array}$} & \multirow[t]{2}{*}{ Type } & \multirow{2}{*}{$\begin{array}{l}\text { HI Velocity } \\
\text { dispersion }\end{array}$} & \multicolumn{2}{|c|}{ Resolution } & \multicolumn{3}{|c|}{ Hi radius } & \multirow{2}{*}{$\begin{array}{c}\text { Distance } \\
\mathrm{Mpc}\end{array}$} & \multirow[t]{2}{*}{ Ref. } \\
\hline & & & & $\begin{array}{c}\text { spectral } \\
\mathrm{km} \mathrm{s}^{-1}\end{array}$ & $\begin{array}{c}\text { spatial } \\
\text { kpc }\end{array}$ & $R_{25}$ & $\mathrm{kpc}$ & $\theta_{\text {maj }}$ & & \\
\hline NGC 4244 & $84.5^{c}$ & Scd & constant $^{d}$ & $5.2^{a}$ & 0.17 & 630 & 11.0 & 2.0 & 3.6 & Olling (1996) \\
\hline ESO142-G24 & $90^{b}$ & Scd & constant $^{e}$ & $6.6^{a}$ & 3.8 & 19.0 & 1.6 & 5.0 & 26.6 & Kregel et al. (2004) \\
\hline ESO157-G18 & $90^{b}$ & Scd & constant $^{e}$ & $6.6^{a}$ & 2.6 & 11.1 & 1.3 & 4.3 & 18.1 & Kregel et al. (2004) \\
\hline ESO201-G22 & $90^{b}$ & $\mathrm{Sc}$ & constant $^{e}$ & $6.6^{a}$ & 9.0 & 34.1 & 1.5 & 3.8 & 55.4 & Kregel et al. (2004) \\
\hline ESO240-G11 & $90^{b}$ & $\mathrm{Sc}$ & constant $^{e}$ & $6.6^{a}$ & 1.7 & 41.5 & 1.4 & 24.2 & 38.1 & Kregel et al. (2004) \\
\hline ESO263-G15 & $90^{b}$ & $\mathrm{Sc}$ & constant $^{e}$ & $6.6^{a}$ & 5.1 & 24.8 & 1.5 & 4.8 & 35.2 & Kregel et al. (2004) \\
\hline ESO269-G15 & $90^{b}$ & $\mathrm{Sc}$ & constant $^{e}$ & $6.6^{a}$ & 7.5 & 19.9 & 1.0 & 2.7 & 46.7 & Kregel et al. (2004) \\
\hline ESO321-G10 & $90^{b}$ & $\mathrm{Sa}$ & constant $^{e}$ & $6.6^{a}$ & 7.3 & 14.5 & 1.2 & 2.0 & 41.0 & Kregel et al. (2004) \\
\hline ESO416-G25 & $90^{b}$ & $\mathrm{Sb}$ & constant $^{e}$ & $6.6^{a}$ & 17.7 & 36.7 & 1.6 & 2.1 & 67.0 & Kregel et al. (2004) \\
\hline ESO435-G14 & $90^{b}$ & $\mathrm{Sc}$ & constant $^{e}$ & $6.6^{a}$ & 7.7 & 18.9 & 1.5 & 2.5 & 35.2 & Kregel et al. (2004) \\
\hline ESO435-G25 & $90^{b}$ & $\mathrm{Sc}$ & constant $^{e}$ & $6.6^{a}$ & 2.2 & 36.1 & 1.2 & 16.3 & 32.4 & Kregel et al. (2004) \\
\hline ESO435-G50 & $90^{b}$ & $\mathrm{Sc}$ & constant $^{e}$ & $6.6^{a}$ & 7.1 & 13.1 & 1.4 & 1.9 & 35.2 & Kregel et al. (2004) \\
\hline ESO446-G18 & $90^{b}$ & $\mathrm{Sb}$ & constant $^{e}$ & $6.66^{a}$ & 12.2 & 31.1 & 1.3 & 2.5 & 64.1 & Kregel et al. (2004) \\
\hline ESO564-G27 & $90^{b}$ & $\mathrm{Sc}$ & constant $^{e}$ & $6.66^{a}$ & 10.2 & 28.7 & 1.6 & 2.8 & 29.5 & Kregel et al. (2004) \\
\hline NGC 5170 & $90^{b}$ & $\mathrm{Sc}$ & constant $^{e}$ & $6.66^{a}$ & 7.4 & 31.9 & 1.3 & 4.3 & 20.9 & Kregel et al. (2004) \\
\hline
\end{tabular}

Notes. ${ }^{(a)}$ Hanning smoothed.

${ }^{(b)}$ From LEDA database.

(c) The unwarped inner disk has an inclination of $i=84.5^{\circ}$, outside of which there is a small warp to $i=82.5 \pm 1^{\circ}$, before warping back to the plane of the inner disk at large radii (Olling 1996).

(d) Direct measurement of the velocity dispersion of the broadening of the extreme velocity envelope of the XV map of an edge-on galaxy. Measurements corrected for instrumental broadening, projection and beam-smearing, via Olling's method (see Sect. 2.6).

(e) Iterative envelope fitting of the XV map of an high-inclination galaxy integrated over the galaxy minor axis (Kregel et al. 2004). This method was unable to measure radial structure of the velocity dispersion, as the method did not include beam correction and the galaxies in the sample were observed with very large beams.

the gas rotation along the line-of-sight. Galaxies inclined slightly away from face-on will suffer beam smearing of the gradients in the projected rotation as well as beam smearing of the gradients of the intrinsic velocity dispersion.

From Table 1, it is seen that only 3 of the low-inclination velocity dispersion studies were undertaken with good spectral resolution (channel $F W H M_{v} \lesssim 5 \mathrm{~km} \mathrm{~s}^{-1}$ ). Of these three high spectral resolution studies, only the recent measurements of
NGC 1058 by Petric \& Rupen (2006) had sufficient spatial resolution to sample the HI radius by at least 10 synthesised beam $F W H M$ s $\left(\theta_{\text {maj }}\right)$. The main difference between the observations of Petric \& Rupen (2006) and the other low inclination HI velocity dispersion measurements are that the observations by Petric \& Rupen (2006) were deeper (23 h total integration time on 3 VLA array configurations) and with good spectral resolution. The two low HI inclination velocity dispersion studies that found 
J. C. O'Brien et al.: The dark matter halo shape of edge-on disk galaxies. II.

Table 3. Summary of previous measurements of rotation curves in edge-on galaxies $\left(i>80^{\circ}\right)$.

\begin{tabular}{|c|c|c|c|c|c|c|}
\hline Galaxies & Method & Line & $\begin{array}{r}\text { Resc } \\
\text { spectral } \\
\mathrm{km} \mathrm{s}^{-1}\end{array}$ & $\begin{array}{l}\text { ution } \\
\text { spatial } \\
\text { kpc }\end{array}$ & $\begin{array}{c}\text { Distance } \\
\text { Mpc }\end{array}$ & Reference \\
\hline UGC 7321 & Peak Flux $^{a}$ & HI & 5.2 & 0.8 & 10 & Uson \& Matthews (2003) \\
\hline $\begin{array}{l}\text { NGC 891 } \\
\text { NGC } 891^{\ddagger} \\
9 \text { dwarfs } \\
4 \text { spirals } \\
4 \text { LSBs }^{\#}\end{array}$ & $\begin{array}{l}\text { Envelope Tracing }^{b} \\
\text { Envelope Tracing }^{b} \\
\text { Envelope Tracing } \\
\text { Envelope Tracing } \\
\text { Envelope Tracing }^{b}\end{array}$ & $\begin{array}{l}\mathrm{HI} \\
\mathrm{HI} \\
\mathrm{HI} \\
\mathrm{HI} \\
\mathrm{HI}\end{array}$ & $\begin{array}{c}27 \\
33 \\
4-8 \\
20-41 \\
4-8\end{array}$ & $\begin{array}{c}1.7 \\
0.7 \\
0.5-2.8 \\
0.9-2.3 \\
0.7-2.7\end{array}$ & $\begin{array}{c}9.5^{f} \\
9.5^{f} \\
3.4-19 \\
9.5-15.5 \\
5-19\end{array}$ & $\begin{array}{l}\text { Sancisi \& Allen (1979) } \\
\text { Swaters et al. (1997) } \\
\text { Swaters (1999) } \\
\text { Sofue (1996) } \\
\text { de Blok \& Bosma (2002) }\end{array}$ \\
\hline NGC $891^{\dagger}$ & $\begin{array}{l}\text { Fixed } \sigma_{v} \& \text { Parametric } v(R) \mathrm{XV} \\
\text { Modelling }\end{array}$ & $\mathrm{HI}$ & 27 & 1.7 & 9.5 & van der Kruit (1981) \\
\hline NGC 5023 & $\begin{array}{l}\text { Fixed } \sigma_{v} \& \text { Parametric } v(R) \mathrm{XV} \\
\text { Modelling }{ }^{b, c}\end{array}$ & HI & 17 & 0.8 & 7.9 & Bottema et al. (1986) \\
\hline NGC 3079 & Iterative Envelope Tracing ${ }^{b}$ & $\mathrm{CO}$ & 5.2 & 0.12 & 15.5 & Takamiya \& Sofue (2002) \\
\hline 14 spirals & $\begin{array}{l}\text { Fixed } \sigma_{v} \text { 1D Gaussian Envelope } \\
\text { Fitting }^{b}\end{array}$ & HI & 6.6 & $1.7-17.7$ & $18-67$ & Kregel et al. (2004) \\
\hline 24 spirals & $\begin{array}{l}\text { Fixed } \sigma_{v} \text { 2D Gaussian Envelope } \\
\text { Fitting }{ }^{b}\end{array}$ & HI & $5.0-20.1$ & $0.8-4.8$ & $5-33$ & García-Ruiz et al. (2002) \\
\hline 8 spirals $^{\star}$ & Fixed $\sigma_{v}$ XV Modelling & $\mathrm{HI}$ & $6.6-33.0$ & $0.9-9.8$ & $9.5-54^{f}$ & Kregel \& van der Kruit (2004) \\
\hline NGC 4244 & $\begin{array}{l}\text { Corrected Gaussian Envelope } \\
\text { Fitting }^{d}\end{array}$ & HI & 5.2 & 0.17 & 3.6 & Olling (1996) \\
\hline
\end{tabular}

Notes. ${ }^{(a)}$ Corrected for instrumental broadening \& gas velocity dispersion using the "equivalent rectangular measure" (Shane \& Bieger-Smith 1966).

(b) Corrected for instrumental broadening \& gas velocity dispersion by quadratic subtraction, see Eq. (9).

(c) Uses the standard Fall \& Efstathiou (1980) parametric rotation curve form, constant $\sigma_{z}$, and a 2D exponential surface density to model the XV diagram.

(d) Corrected for broadening \& beam-smearing (see Olling's method in Sect. 2.6).

(e) Asymmetric drift correction applied.

(f) Using distance measured by van der Kruit (1981) for NGC 891.

(†) Re-analysis of NGC 891 data observed by Sancisi \& Allen (1979).

(‡) New deeper WSRT NGC 891 data $-144 \mathrm{~h}$.

(\#) Re-analysed subset of the Swaters (1999) dataset.

(^) Includes re-analysed NGC891 data observed by Swaters et al. (1997).

a constant velocity dispersion with radius were only marginally spectrally resolved (channel $F W H M_{v}=8.2 \mathrm{~km} \mathrm{~s}^{-1}$ ), and likewise also marginally spatially resolved $\left(5<R_{\mathrm{HI}} / F W H M_{\theta}<10\right)$.

Of the HI velocity dispersion measurements of high inclination galaxies (see Table 2), only the study of NGC 4244 (Olling $1996)$ has the spatial resolution ( $100 \mathrm{pc})$ to investigate the radial velocity dispersion on scales relevant to common galactic structure (star formation, spiral density waves, bars). However, Olling's method (1996) used only part of the HI emission distribution, and may not be accurate (see Sect. 2.6). The other high inclination velocity dispersion measurements obtained by Kregel et al. (2004) used an HI velocity dispersion fit that was held fixed with radius, consequently no information was obtained about the radial variability of the velocity dispersion (see the discussion of the method in Sect. 2.3).

As a result, very little is known about the velocity dispersion of the HI in the ISM. The dependence of the HI velocity dispersion on galaxy mass, surface brightness, star formation rate, and density variations such as spiral arms or bars is unknown. Proper modelling of the full HI emission cube gives the radial distribution of surface density, rotation and velocity dispersion. This approach could be expanded to also model the HI transparency of the HI emission distribution of a galaxy, whether edge-on or inclined.

In the past, low telescope resolution and deprojection issues made such studies nonviable. In this paper, we show that it is possible to accurately perform the deprojection required to measure the velocity dispersion, rotation and surface density as functions of radius in edge-on galaxies. The method is also applicable to galaxies of moderate inclination. The edge-on orientation of galaxies in our study is only required to minimise the uncertainty of the measured vertical gas flaring, which is required to determine the dark halo flattening. If fitting galaxies less inclined than edge-on, the inclination must be accurately fitted at all radii, and the galaxy must not be too close to face-on or else the line-of-sight velocity will be not be sufficiently resolved to model the galaxy rotation. Application of our new HI emission modelling method to nearby non-edge-on spiral galaxies, would allow investigation of the velocity dispersion, transparency, temperature and density structure of neutral hydrogen in the ISM of a large sample of galaxies. This could explain how this smallscale ISM structure varies with the mass and star formation rate of spiral galaxies.

Many methods have been devised to measure the rotation curves of edge-on galaxies. In Table 3, we display the previous rotation curve measurements of edge-on galaxies, showing the method used and the resolution of the observations. In the next section, we will briefly review these methods, and the few previous methods to measure the gas velocity dispersion and surface density, before presenting our new method in Sect. 3 .

In the Paper III of this series we will present the results of applying this new method to the HI observations of the edge-on galaxy sample presented in Paper I. In that paper, we will paper also derive the radial flaring of the HI layer of these galaxies. 
A determination of the flattening of the dark halo of one galaxy in our sample, UGC 7321, will be presented in Paper IV.

\section{Methods to extract rotation curves for edge-on galaxies}

High accuracy rotation curves and deprojected HI surface densities can be difficult to measure accurately from HI observations of edge-on disk galaxies, due to the projection of the velocity profiles from a range of radii into a single line-of-sight velocity profile. We will first discuss the methods that have been used previously and the accuracy of their results. None of these methods independently determine the HI gas velocity dispersion from the data. These methods provided a useful starting point from which to develop a new method that incorporates the determination of velocity dispersion directly from the observations.

\subsection{The peak flux and envelope tracing method for measuring the rotation curve}

Most previous measurements of the HI rotation curves from edge-on galaxies use the shape of the outer envelope of the position velocity diagram, using either the Peak Flux method (Rubin et al. 1985; Mathewson et al. 1992; Uson \& Matthews 2003) or the Envelope Tracing method (Sancisi \& Allen 1979; Sofue 1996; García-Ruiz et al. 2002). The Peak Flux method adopts the line-of-sight velocity at the maximum flux along each slice of the XV diagram as the rotation velocity at the line of nodes in that sightline. The Envelope Tracing method instead uses the line-of-sight velocity at a nominal level, e.g. $20 \%$ of the peak flux, on the outer velocity side of each slice through the XV diagram. Both these methods also involve application of a correction to the measured velocities, to correct for the combined line broadening due to the instrumental broadening and the unknown intrinsic velocity dispersion of the HI.

The main problem with such methods is that the flux level of the actual rotation velocity at any one radius is dependent on three different unknown radial functions: the actual rotation curve, the deprojected HI surface density and the HI velocity dispersion. The peak flux method underestimates the rotation, while the envelope tracing method tends to adopt the velocity at a flux level dependent on the signal-to-noise of the observations. Too low a flux level will overestimate the rotation, while the converse is true, if too high a level is chosen.

The accuracy of derived rotation velocities also depends on the accuracy of the adopted gaseous velocity dispersion used in the velocity broadening correction of the measured velocity. Users typically adopt a nominal radially invariant value of $6-10 \mathrm{~km} \mathrm{~s}^{-1}$ from direct vertical HI velocity dispersion measurements of normal face-on disk galaxies (van der Kruit \& Shostak 1984; Lo et al. 1993).

Beam-smearing also tends to increase the velocity of the outer envelope, while lowering the velocity of the peak flux of slices in the inner disk regions, where the radial gradient of rotation is larger. Beam smearing is a significant problem in most HI rotation curve studies of edge-on spiral galaxies as few measurements use observations with a resolution greater than 1-2 kpc (see Table 3).

The shape of the extreme velocity edge of the XV diagram is constructed from superposition of gas all along the line-of-sight, including gas from the line-of-nodes to the edge of the disk. The gas at each location has an intrinsic velocity dispersion that may be variable with radius. The edge-on orientation of the disk

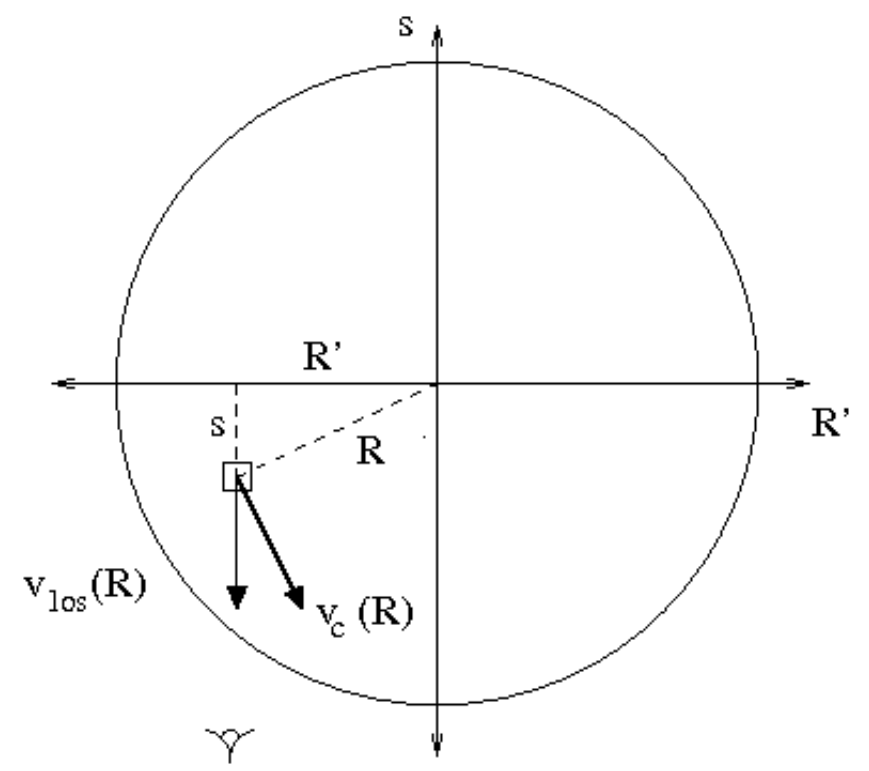

Fig. 1. Diagram of galaxy coordinate system used to model the $X V$ gas distribution in Eq. (6). This shows the major axis $R^{\prime}$, depth along the line-of-sight $s$, and the projection of galaxy rotation along the line-ofsight towards the observer.

projects all of these radial velocity profiles onto a single line-ofsight velocity profile at each line-of-sight position through the disk (see for example Fig. 1 of Kregel \& van der Kruit 2004). Due to this projection effect it can be inferred that the envelope tracing method would fail to accurately measure the rotation curve, even if it were used with a correct HI velocity dispersion, high-signal-to-noise and high resolution data.

Consequently, the rotation curve derived with these two methods may exhibit an incorrect shape regardless of the signalto-noise and spatial resolution of the observations. For example, an adopted HI velocity dispersion say with an dispersion uncertainty of $3 \mathrm{~km} \mathrm{~s}^{-1}$, will cause a rotation curve uncertainty of $7 \mathrm{~km} \mathrm{~s}^{-1}$ due to the velocity dispersion error alone. As the HI velocity dispersion uncertainty is not known, if it is adopted from low resolution face-on measurements of other spiral galaxies, the actual rotation curve error could be larger. Consequently many high inclination rotation curve measurement are likely to be in error by between $7-20 \mathrm{~km} \mathrm{~s}^{-1}$, when the effects of projection and beam smearing are included. Worse still, if the HI were partially opaque, the inner disk rotation may be further underestimated.

\subsection{The iterative envelope tracing method for measuring the rotation curve}

In 2002, Takamiya \& Sofue (2002) developed the iterative envelope tracing method. This improves on the standard envelope tracing method by automatically adjusting the initial envelope tracing rotation curve, until the envelope tracing fit to a model $\mathrm{XV}$ distribution built with this model rotation curve matches the original envelope tracing rotation curve fit to the observed $\mathrm{XV}$ distribution. To test the rotation curve fit derived with the standard envelope tracing method, Takamiya \& Sofue (2002) used this fit to build a model XV distribution which they also fitted with the envelope tracing method. By comparing the two fits, and adjusting the model rotation curve accordingly, they incremented the model rotation curve until it generated an XV diagram that produced the same envelope tracing fit as the observed 
XV diagram. The initial fit $v_{\text {fit }, 0}(R)$ to the observed XV diagram was used as the first model rotation curve $v_{\text {model, } 1}(R)$, with which the first model $\mathrm{XV}$ diagram $\mathrm{XV}_{1}$ was built and a new envelope tracing fit $v_{\mathrm{fit}, 1}(R)$ obtained. This was used to estimate a revised rotation curve model $v_{\text {model, } 2}(R)$ by comparing the new fit $v_{\text {fit, }, 1}(R)$ to the original fit $v_{\mathrm{fit}, 0}(R)$. At each radius where the difference was greater than some tolerance $v_{\text {crit }}$, the model rotation curve was incremented by the difference $v_{\mathrm{fit}, 0}(R)-v_{\mathrm{fit}, 1}(R)$, thereby forming a revised model rotation curve $v_{\text {model, } 2}(R)$. A new model $\mathrm{XV}$ diagram was built with the revised model rotation curve and the process was iterated, as shown below in Eqs. (2)-(4), until the fit $v_{\text {fit }, n}(R)$ matched the original fit $v_{\text {fit }, 0}(R)$. Whereupon the model rotation curve $v_{\text {model }, n}(R)$ was taken to be the best model for the observations.

$$
\begin{aligned}
& \Delta v_{\text {fit }, n}(R)=v_{\text {fit }, 0}(R)-v_{\text {fit }, n}(R) \\
& v_{\text {model }, n+1}(R)= v_{\text {fit }, 0}(R)+\Delta v_{\text {fit }, n}(R) \\
& \text { if } \Delta v_{\text {fit }, n}(R)>v_{\text {crit }}, \\
& v_{\text {model }, n+1}(R)= v_{\text {fit }, 0}(R) \\
& \text { if } \Delta v_{\text {fit }, n}(R) \leq v_{\text {crit } .}
\end{aligned}
$$

Takamiya \& Sofue employed several assumptions to build each model XV diagram. Firstly, the radial gas surface density was approximated by the gas surface density along the major axis, which was derived from the observed XV diagram by integrating over velocity. Secondly, the gas velocity dispersion was assumed to be isotropic and isothermal in $R$ and $z$ with an adopted a value of $10 \mathrm{~km} \mathrm{~s}^{-1}$, which is typically observed in HI in spiral disks (Shostak \& van der Kruit 1984; van der Kruit \& Shostak 1984). In the $z$ direction the HI surface density was approximated by a $\operatorname{sech}^{2}$ model with a constant scale height of $100 \mathrm{pc}$. To simulate the observing process, each model XV cube was then convolved by the spatial and spectral resolution of the telescope, and integrated over $z$ to form the model XV map.

Takamiya \& Sofue (2002) applied this method and the earlier peak flux and envelope tracing methods to optical emission spectra and $\mathrm{CO}(J=1-0)$ observations with the Nobeyama Millimetre array on the edge-on galaxy NGC 3079, and four moderately inclined galaxies: NGC 4536, NGC 4254, NGC 4419, NGC 4501. They found that both the envelope tracing and peak flux methods underestimated the rotation velocities in the inner regions of their galaxies, with the peak flux method being in error by up to 50-100\%. Furthermore at outer radii with low signal-to-noise, the derived rotation velocities exhibited dramatic scatter of $\pm 20-100 \mathrm{~km} \mathrm{~s}^{-1}$ (see Fig. 4a of Takamiya \& Sofue 2002), probably because the $3 \sigma$ noise level was above the $20 \%$ flux level used for envelope tracing.

Sofue et al. (2003) applied the method to CO observations of a sample of moderately inclined galaxies $\left(25^{\circ}<i<74^{\circ}\right)$, but this time with a small difference. Instead of fitting the XV diagrams at a single fractional flux level, they fitted the lineof-sight velocity at a series of flux levels $l=20,40,60,80$ and $100 \%$, and replaced Eq. (2) by

$\Delta v_{\mathrm{fit}, n}(R)=\sum_{l} g_{l} \times\left[v_{\mathrm{fit}, 0}^{l}(R)-v_{\mathrm{fit}, n}^{l}(R)\right]$

where $g_{l}$ is a set of weights satisfying $\sum_{l} g_{l}=1$, and usually evenly weighted, except when low flux levels are biased by noise in sightlines through the XV diagram with low signal-tonoise. The iterative envelope tracing method is an improvement because it checks the veracity of the measured rotation curve. However, substantial flaws remain: it uses an incorrect radial gas surface density, and an assumed constant gas velocity dispersion.

\subsection{The fixed velocity dispersion, 1D Gaussian envelope fitting method for measuring the rotation curve and approximating the gas velocity dispersion}

García-Ruiz et al. (2002) improved on the standard Gaussian model of the full outer envelope of the XV diagram, by performing a series of three-parameter Gaussian fits to the outer envelope of the velocity profile in each sightline. The first channel on the outer velocity envelope with flux $>3 \sigma$ was the outermost velocity channel used in each Gaussian fit. For the first Gaussian fit, this outer channel and the two lower channels were included in the profile sample. Subsequent fits were performed, each time adding the next lower velocity channel, until the velocity channel with peak flux was reached. The centre of the Gaussian with the smallest reduced $\chi^{2}$ was retained for that sightline.

Kregel et al. (2004) applied the fixed velocity dispersion 1D Gaussian envelope fitting method to 14 galaxies using an radially constant velocity dispersion of $10 \mathrm{~km} \mathrm{~s}^{-1}$. Successful fits were obtained for 11 galaxies using this HI velocity dispersion, however the remaining three galaxies required the velocity dispersion to be reduced to $6-7 \mathrm{~km} \mathrm{~s}^{-1}$ to achieve a converging fit. However, the large beam size of the observations $\left(1.7 \lesssim F W H M_{\theta} \lesssim 18 \mathrm{kpc}\right)$ masked any small scale variations in the rotation speed. Only 3 of the galaxies in their sample have a HI radius greater than 5 beamwidths. (See Table 2.) For two of the three galaxies, which required smaller HI velocity dispersions, the radius was spanned by only 2.5 synthesized beams. Consequently, due to the low spatial resolution, they were unable to measure any radial structure of the velocity dispersion.

We show in Sect. 4.3 that even an intrinsically constant gas velocity dispersion should appear broadened near the Galaxy centre due to beam smearing - typically by $\geq 50-100 \%$, if $F W H M_{\theta}>1 \mathrm{kpc}$. This beam smearing effect would cause the $\chi^{2}$ error of the velocity dispersion fits at inner radii to dramatically increase as the XV slice bears progressively less resemblance to a sum of Gaussians.

An advantage of this method is that it could be extended to measure the gas velocity dispersion as a function of radius, by allowing the velocity dispersion to be a free parameter in the Gaussian envelope fits, and correcting the measured velocity dispersion for instrumental broadening using Eq. (9).

\subsection{The fixed velocity dispersion, 2D Gaussian envelope fitting method for measuring the rotation curve}

To attempt to correct the fixed velocity dispersion 1D Gaussian envelope fitting method (Sect. 2.3), García-Ruiz et al. (2002) applied the same series of fixed dispersion Gaussian fits to slices along the radial $R^{\prime}$ direction of the XV diagram; i.e., in addition to fitting at velocity profile at each major axis position $R^{\prime}$, they also fitted major axis $R^{\prime}$ profiles of the flux in each velocity channel $v$. The width of the outer envelope in the $R^{\prime}$ direction was determined by the $F W H M_{\theta}$ of the synthesised beam and the unknown gradient of the rotation curve. They measured this spatial dispersion along the $R^{\prime}$ profile at the systemic velocity, $v_{\text {los }}=v_{\text {sys }}$, of each galaxy, and held this value fixed when Gaussian fitting the $R^{\prime}$ profile of each channel. The rotation velocity at each radius was taken to be the minimum of the rotation velocities measured with each method.

Using an adopted HI velocity dispersion of $8 \mathrm{~km} \mathrm{~s}^{-1}$, García-Ruiz et al. (2002) applied this method to measure the rotation curves of 24 edge-on $\left(i>80^{\circ}\right.$ ) galaxies (see Table 3 ). Beam-smearing correction was important for this sample as most of their observations had a FWHM beam of 1-2 kpc, and most of 
Kinematic fit
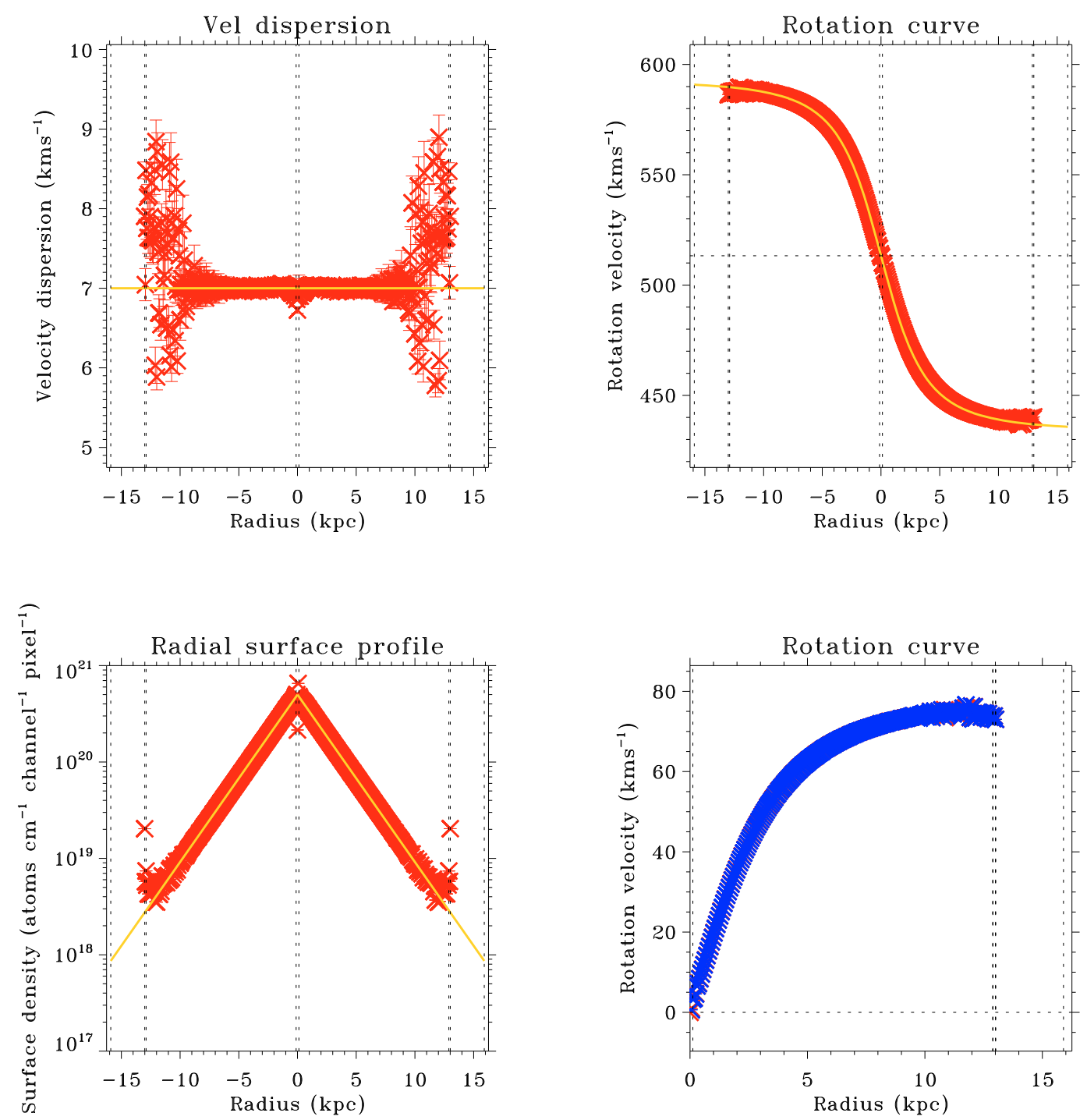

Fig. 2. This is a derived gas kinematics fitted from a raw synthetic XV map (i.e. not spatially smeared by the telescope beam and without noise) using the radial decomposition method. The plots of gas velocity dispersion, rotation and deprojected surface density show the actual synthetic galaxy kinematic as a grey (yellow) line and measured fits in (red) crosses. The plot in the bottom right hand corner shows both sides of the measured rotation curve (in the color version the receding side is plotted first in red and the approaching side on top in blue). Error bars are shown on all fits, though in most cases they are smaller than the plot symbol. Subsequent similar figures in this paper will use the same plotting scheme.

the galaxies in their sample were moderately resolved spatially with $5 \leq R_{\mathrm{HI}} / F W H M_{\theta}<10$.

\subsection{The fixed velocity dispersion, $X V$ modelling method for measuring the rotation curve and deprojected HI surface density}

The basic approach of this method is to directly fit the full observed distribution in the $\mathrm{XV}$ plane to derive the rotation curve and the radial HI distribution. It was first applied by van der Kruit (1981) and was further developed by Kregel \& van der Kruit (2004). We regard this method as the most powerful approach. Kregel \& van der Kruit (2004) model the full observed XV diagram as a set of concentric, coplanar rings, by progressively measuring the rotation $v(R)$ from the outside in, or as they call it the "onion-peeling" approach.

For a diagram of the coordinate system we refer to Fig. 1. By modelling the HI layer as an axisymmetric, transparent isothermal sheet in pure circular rotation, the velocity profile probed by sight lines through the $\mathrm{XV}$ diagram is

$$
\begin{aligned}
f_{\mathrm{HI}}\left(R^{\prime}, v\right)= & \frac{1}{\sigma_{v, \text { tot }} \sqrt{2 \pi}} \int \mathrm{d} s \Sigma_{\mathrm{HI}}(R) \\
& \times \exp \left(-\frac{\left[v-v_{\mathrm{c}}(R)\left(R^{\prime} / R\right)\right]^{2}}{2 \sigma_{v, \text { tot }}^{2}}\right),
\end{aligned}
$$

where

$v_{\mathrm{los}}(R)=\left(\frac{R^{\prime}}{R}\right) v_{\mathrm{c}}(R), \quad R=\sqrt{R^{\prime 2}+s^{2}}$

and $R^{\prime}$ is the projected radius on the sky, $s$ the depth along the line of sight (i.e. $s=0$ at the line-of-nodes), $R$ the galactocentric radius, $\Sigma_{\mathrm{HI}}(R)$ the radial face-on surface density profile and $\sigma_{v \text {,tot }}(R)$ the total velocity dispersion; see Eq. (9). $v_{\mathrm{c}}(R)$ is the rotation velocity and $v_{\mathrm{los}}(R)$ is the projection of the rotation 


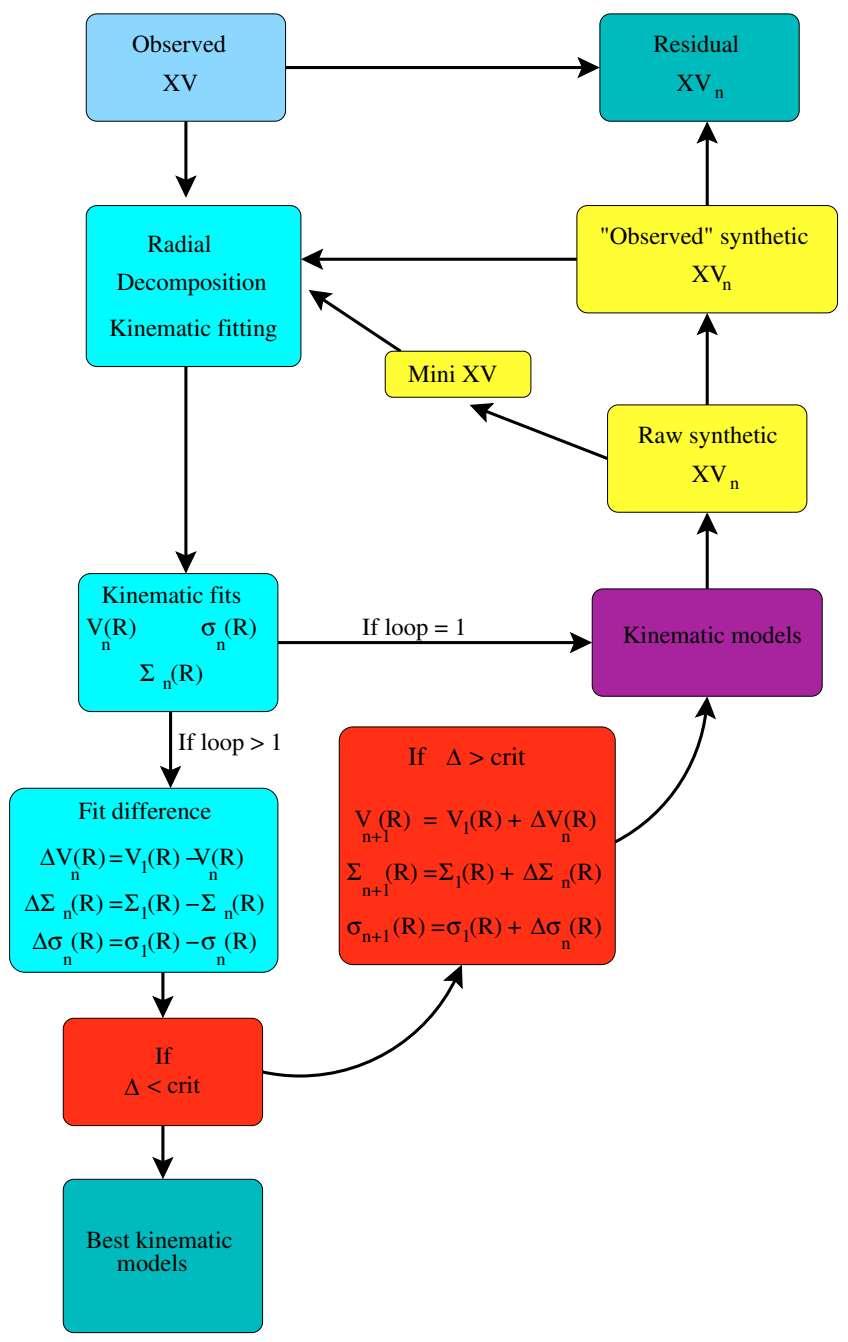

Fig. 3. Flow chart showing the iterative radial decomposition method.

velocity onto the line-of-sight. This method assumes a constant HI velocity dispersion $\sigma_{\mathrm{v}, \mathrm{HI}}$ of $10 \mathrm{~km} \mathrm{~s}^{-1}$. The face-on surface density $\Sigma_{\mathrm{HI}}(R)$ was derived independently using the Warmels' (1988) method, which uses the Lucy algorithm to invert an Abel integral (e.g. Binney \& Tremaine 1987, Eqs. (1B)-(59b)).

At each sightline the HI surface density and rotation on the line of nodes was fitted using a single Gaussian. By starting at the outermost projected radius $R^{\prime}=R_{\text {outer }}^{\prime}$ and using at least 4 independent data points above a nominal noise flux level, the rotation velocity was determined by a Gaussian fit to the outer envelope. The next inwards profile at $R^{\prime}=\left(R_{\text {outer }}^{\prime}-\Delta R^{\prime}\right)$ was then fitted simultaneously for $\left(R_{\text {outer }}^{\prime}-\Delta R^{\prime}\right)$ and $R^{\prime}$, where $\Delta R^{\prime}$ denoted the annulus width, which was set to the pixel size of the observations. An initial estimate of the value of the rotational velocity determined in the previous fit was used at $R_{\text {outer }}^{\prime}$, while at the line-of-nodes $\left(R_{\text {outer }}^{\prime}-\Delta R^{\prime}\right)$ the 1D Gaussian envelope tracing value was measured. Each line profile was fitted as a set of fixed dispersion Gaussians with flux determined from the derived surface density, yielding the rotation velocity at the line-of-nodes $v\left(R=R^{\prime}\right)$ and new values for the rotation velocities (measured in projection) of all high radii annuli in the sight line.

The procedure was repeated, progressively determining the rotation velocity at all radii inwards until the centre was reached, and then the same method was applied to the other side of the galaxy. In this way the fitting algorithm does not become ill-constrained, as the number of free parameters increases at the same rate as the initial estimates of the fit parameters.

In addition to more accurately determining the rotation velocity by modelling the full profile through each sight line of the XV diagram, Kregel \& van der Kruit (2004) noted that this method also successfully corrects the rotation velocity for the effects of beam smearing. This was done by first building a model mini XV diagram comprising sufficient sightlines adjacent and outwards of $R^{\prime}$ to span at least the beam $F W H M_{\theta}$ using Eq. (6), and convolving by the instrumental broadening and any Hanning taper (if applied). The mini XV diagram is then convolved spatially by the known beam $F W H M_{\theta}$. The velocity profile of interest, can then be corrected for the modelled beam smearing effects determined from the mini XV diagram, prior to Gaussian fitting.

The best fit rotation velocity at each radius was then determined by $\chi^{2}$ minimization using the "amoeba" (Press et al. 1992) implementation of the simplex method (Nelder \& Mead 1965). This easily handles a large number of free parameters and does not require the derivatives of the model with respect to those parameters (a computation-intensive calculation). Errors were estimated using Monte Carlo simulations.

Kregel \& van der Kruit (2004) applied this method to the HI observations of 14 edge-on galaxies. These comprised 13 of the galaxies in the Kregel et al. (2004) sample and HI observations of NGC 891 by Swaters et al. (1997). The fitting method successfully converged to sensible results for all the galaxies, except five galaxies with low peak signal-to-noise of $S / N<15$, and one galaxy NGC 5529 which displays a pronounced warp of the disk plane. The resolution of the galaxies with successful fits are shown in Table 3. Despite the large beam sizes $\left(1 \lesssim F W H M_{\theta} \lesssim 10\right)$, two of these successfully fitted galaxies (NGC 891 and ESO 435-G25) that were well sampled by the beam with $R_{\mathrm{HI}} / F W H M_{\theta}>10$, while four had moderate sampling with $5 \leq R_{\mathrm{HI}} / F W H M_{\theta}<10$, and two were successfully fitted despite poor sampling of $R_{\mathrm{HI}} / F W H M_{\theta}<5$.

For four galaxies with higher $S / N$ observations, Kregel \& van der Kruit (2004) also succeeded in directly fitting the surface density by allowing it to be a free parameter in the Gaussian fits. They compared the rotation curve and surface densities derived from their method with the rotation curve derived from standard envelope tracing method and the surface density derived from the Warmels' (1988) deprojection method. Each method was applied to a simulated HI XV diagram of NGC 2403, built with the "true" rotation curve and surface density measured by Fraternali et al. (2002) using extensive 3D modelling, showing that the fixed velocity dispersion XV modelling method correctly measures both the rotation curve and the deprojected surface density. Kregel \& van der Kruit's surface density deprojection method is a significant improvement on that of (Warmels 1988), which was unable to recover the surface density variations on radial scales smaller than the $F W H M_{\theta}$ of the synthesised beam.

\subsection{Direct outer envelope fit method for measuring the velocity dispersion, rotation curve and deprojected HI surface density}

Olling (1996) used the simple outer envelope velocity dispersion fit, but then endeavoured to correct the measured broadening for the major effects of beam-smearing and velocity profile projection in addition to instrumental broadening. His correction method (detailed in Appendix A of Olling 1996) calculated the effect of the projection given in Eq. (6) to determine how to correct the measured direct Gaussian fit to the outer velocity 

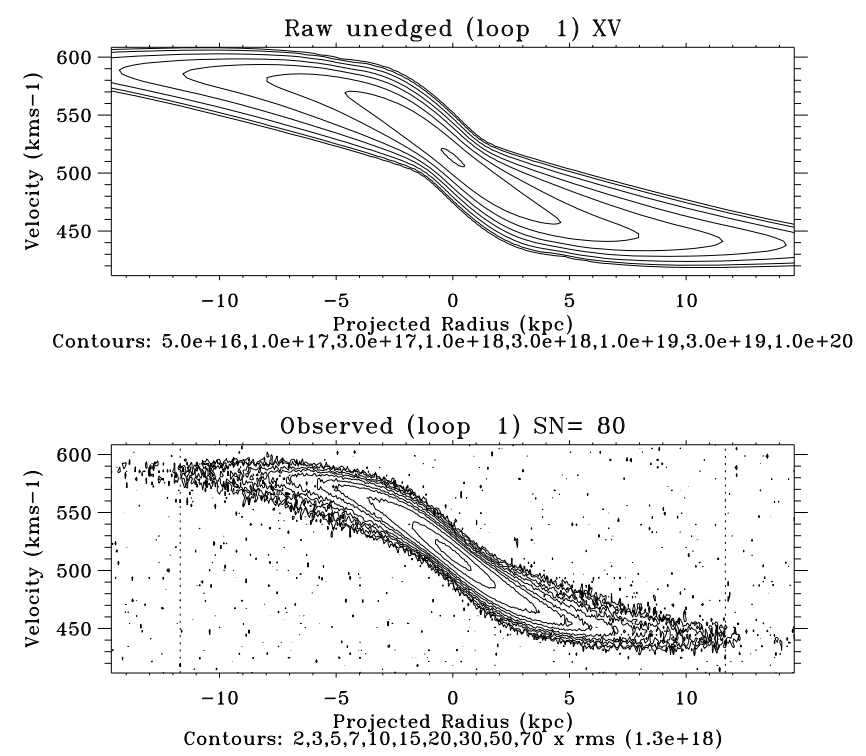
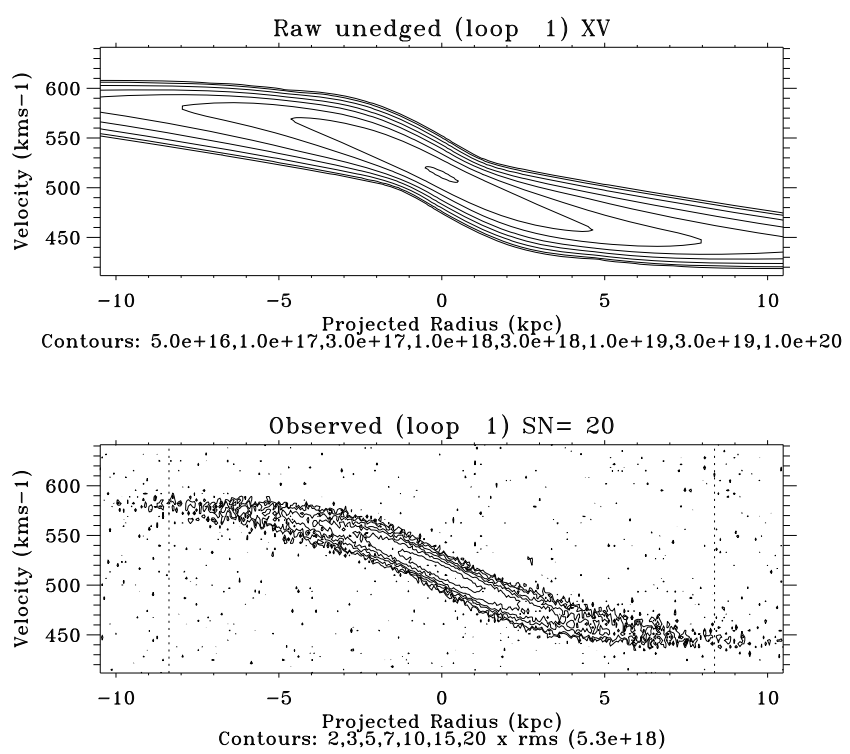

Fig. 4a. Actual and observed XV maps of the synthetic galaxy. This has the properties illustrated by the grey (yellow) lines in Figs. 4b and c. The top XV diagrams show the actual XV HI distribution, while the lower plot is the XV diagram as observed to detection limits corresponding to peak noise-to-noise $(S / N)$ of 80 and 20, each with the same telescope $F W H M$ of $300 \mathrm{pc}$. The contours in the upper panels are (in units of $10^{17}$ atoms cm ${ }^{-1}$ pixel $^{-1}$ ) at $0.5,1,3,10,30,100,300$ and 1000 and in the lower panels at 2, 3, 5, 7, 10, 15, 20, 30, 50 and 70 times the rms noise in the channel maps. The effect of increased noise shows clearly the reduced fraction of the actual XV diagram that is detected. The vertical dashed lines on the observed XV maps mark the outermost radius with sufficient flux above $2 \sigma$ to allow kinematic fitting. Note that the scales are different in the plots on the right and the left, because of changing observable extent for different $S / N$.

envelope of each slice of the XV diagram. By approximating the Gaussian in Eq. (6) as a parabola, and integrating over several velocity channels around the line-of-node $\left(R=R^{\prime}\right)$, Olling derived an analytical approximation of the velocity profile around the line-of-nodes to be (Eq. (A5) of Olling 1996):

$$
\begin{aligned}
I\left(R^{\prime}, v\right) \approx & \frac{\epsilon_{m} K \Delta v \Sigma_{\mathrm{HI}}(R)}{\sigma_{v}(R) \sqrt{2 \pi}} e^{-\frac{1}{2}\left(\frac{W(R)}{\sigma_{v}(R)}\right)^{2}} \times\left(2-\frac{\epsilon_{m}^{2} W(R) v_{\mathrm{c}}(R)}{3 \sigma_{v}(R)^{2}} \frac{R^{\prime} \epsilon_{m}^{2}}{3}\right. \\
& \left.\times\left[\frac{a W(R)}{\sigma_{v}(R)^{2}}+b\left(\frac{W(R)^{2}}{\sigma_{v}(R)^{2}}-1\right)+\frac{c}{\Sigma_{\mathrm{HI}}(R)}\right]\right)
\end{aligned}
$$

where the kinematics (i.e. $v_{\mathrm{c}}(R), \sigma_{v}(R)$ and $\left.\Sigma_{\mathrm{HI}}(R)\right)$ were evaluated at the line-of-nodes $R=R^{\prime}$, and $W(R)=v-v_{\mathrm{c}}(R) . \Delta v$ is the channel width, $K, a, b$ and $c$ are constants, $\epsilon_{m}=s_{\max } / R^{\prime}$, and $s$ is the depth along the line-of-sight (see the schematic of the coordinate system in Fig. 1). The first term, 2, results from gas on the line-of-nodes. The second term arises due to material close to the major axis, and the last 3 terms arise due to radial gradients in the rotation velocity, gas velocity dispersion and surface density, respectively.

Olling (1996) applied this method to one galaxy, NGC 4244, which was observed at high spatial and spectral resolution with a $F W H M_{\theta}$ of $170 \mathrm{pc}$ and a $F W H M$ channel width of $5.2 \mathrm{~km} \mathrm{~s}^{-1}$. Although NGC 4244 is a small Scd galaxy with maximum HI radius of $11 \mathrm{kpc}$, the high spatial resolution allowed the radial HI structure to be sampled by 63 FWHM beams on either side of the galaxy centre (see Table 2). By testing the above method on XV simulations of NGC 4244, he found that Eq. (8) was sufficient to recover the kinematics in the outer galaxy. However, within $6 \mathrm{kpc}$ from the galaxy centre, beam-smearing became a problem. To derive the gas properties in the inner disk consistent with the beam smearing, Olling (1996) used simulations of NGC 4244 to determine an empirical correction to the kinematics measured with Eq. (8). In order to simulate the XV diagram of NGC 4244, Olling had to estimate the intrinsic kinematics, as the derived corrections depend on the intrinsic XV diagram as well as the resolution of the observations. With this method Olling found that the velocity dispersion of NGC 4244 was roughly constant at $8.5 \pm 2 \mathrm{~km} \mathrm{~s}^{-1}$. Unlike the HI velocity dispersion measurements of face-on galaxies which mostly decline with radius (see Table 1), Olling's analysis of NGC 4244 shows no radial structure in the velocity dispersion despite being measured on scales of $\sim 100 \mathrm{pc}$.

\section{The radial decomposition XV modelling method}

We developed the radial decomposition XV modelling method to more accurately measure the rotation, velocity dispersion and deprojected surface density of HI disks. Like the Kregel \& van der Kruit (2004) method it operates by fitting the galaxy from the outer radii inwards - "onion-peeling" as they called it. The radial decomposition method works by progressively fitting each sightline of the observed XV diagram, starting at the outermost edge, to measure the rotation velocity, surface density and velocity dispersion. The basic method is essentially the same as in Kregel \& van der Kruit (2004), however we also fit the HI velocity dispersion, and extend the method to iterate further if required. By progressing inwards, the method uses the measured kinematics of outer radii to isolate and fit only the velocity profile of the line of nodes gas along each sightline.

This allows one to accurately fit the rotation, velocity dispersion and surface density as functions of galactocentric radius despite the edge-on orientation of the galaxy. The measured velocity dispersion is corrected for the broadening due to the finite spectral resolution and the derived HI velocity dispersion at each radius is used in the measurement of all inner annuli.

The method works by modelling the radial and velocity distribution of each slice through an edge-on galaxy that has been integrated over the vertical axis $z$. This requires the usual assumptions of circular motion, azimuthal symmetry, isotropy of the HI velocity dispersion tensor. To maximise the peak signalto-noise of the XV map, the map is formed by integrating over 
Kinematic fit
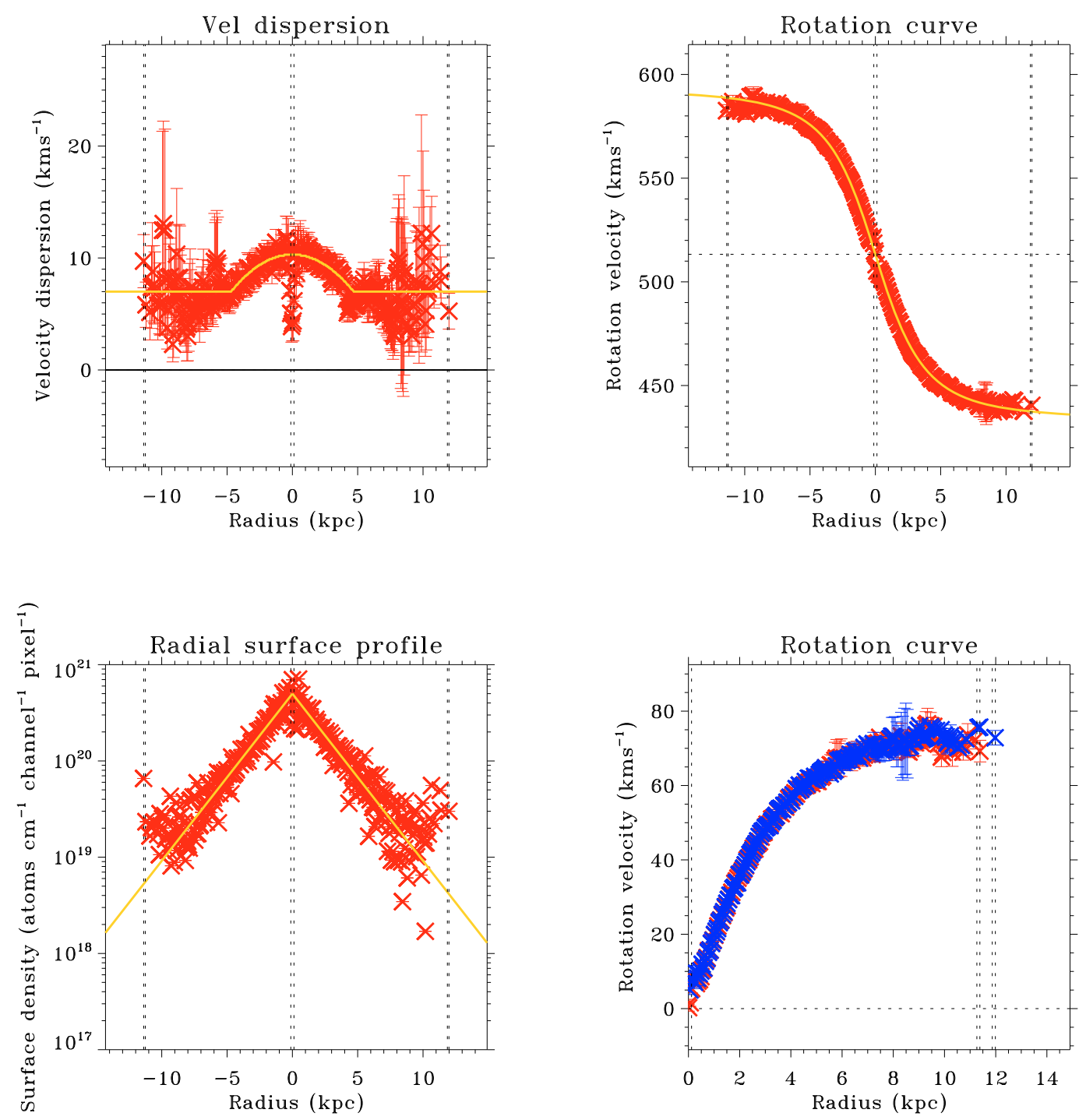

Fig. 4b. Fitted kinematics measured from an XV diagram observed with a telescope beam of $F W H M_{\theta}=300$ pc and to a peak signal-to-noise of 80 . Beam-smearing correction is not necessary at this high resolution, as the scatter due to beam-smearing is much smaller than the scatter due to noise. This figure and the plots in Fig. $4 \mathrm{c}$ demonstrate the effect of noise on the measured kinematics. To reduce the scatter we have averaged the measurements using a evenly weighted 5 pixel wide bin after fitting the whole XV diagram via the radial decomposition method. The outermost dashed lines indicate the maximum radius $R_{\max }$ with sufficient flux to be fitted. The other dashed vertical lines mark a beam $H W H M$ from $R_{\max }$ on either side, and a $H W H M$ from the galaxy centre.

the full range $z$ spanned by the edge-on galaxy in the vertical direction. This requires that the velocity dispersion is vertically isothermal and that the rotation is cylindrical. In future studies, the assumption of HI isothermality should be tested by applying the radial decomposition XV modelling method to deep observations of both low and high lattitude HI observed at high spatial resolution. This would determine whether the velocity dispersion (and rotation) varies with height above the galactic plane. Although the HI in some deeply observed galaxies like NGC 891 (Fraternali et al. 2005) does show a rotational lag in the Hi layer a few kpc above the galactic plane, our data is for the most part confined to a thin layer of $F W H M<2 \mathrm{kpc}$, so the weighted effect of the high-latitude lag on our analysis is unlikely to be significant.

Although the method will not detect spatial asymmetries in the gas surface density, such as might be expected from bars or spiral waves, it will detect radial annuli devoid of HI, or a central $\mathrm{HI}$ hole, as the surface density is modelled as $\Sigma_{\mathrm{HI}}(R)$.

The method fits the radial kinematics by modelling each sightline through the XV diagram as a cut through a superposition of annular rings, in which the gas at each radius exhibits a Gaussian velocity profile described by the radial gas kinematics seen in projection according to Eq. (6). Each slice comprises gas at a range of radial bins, with the gas in each radial bin in the slice forming a Gaussian profile

- centred at the rotation velocity seen in projection, $v_{\text {los }}$ $\left(v_{\mathrm{los}}=v(R)\right.$ along the line-of-nodes, $v_{\mathrm{los}}<v(R)$ for radii outside the nodal radius);

- broadened to a velocity $F W H M$ due to the effect of intrinsic velocity dispersion and the instrumental resolution; $F W H M_{\mathrm{v}, \text { tot }}$ and 
Kinematic fit
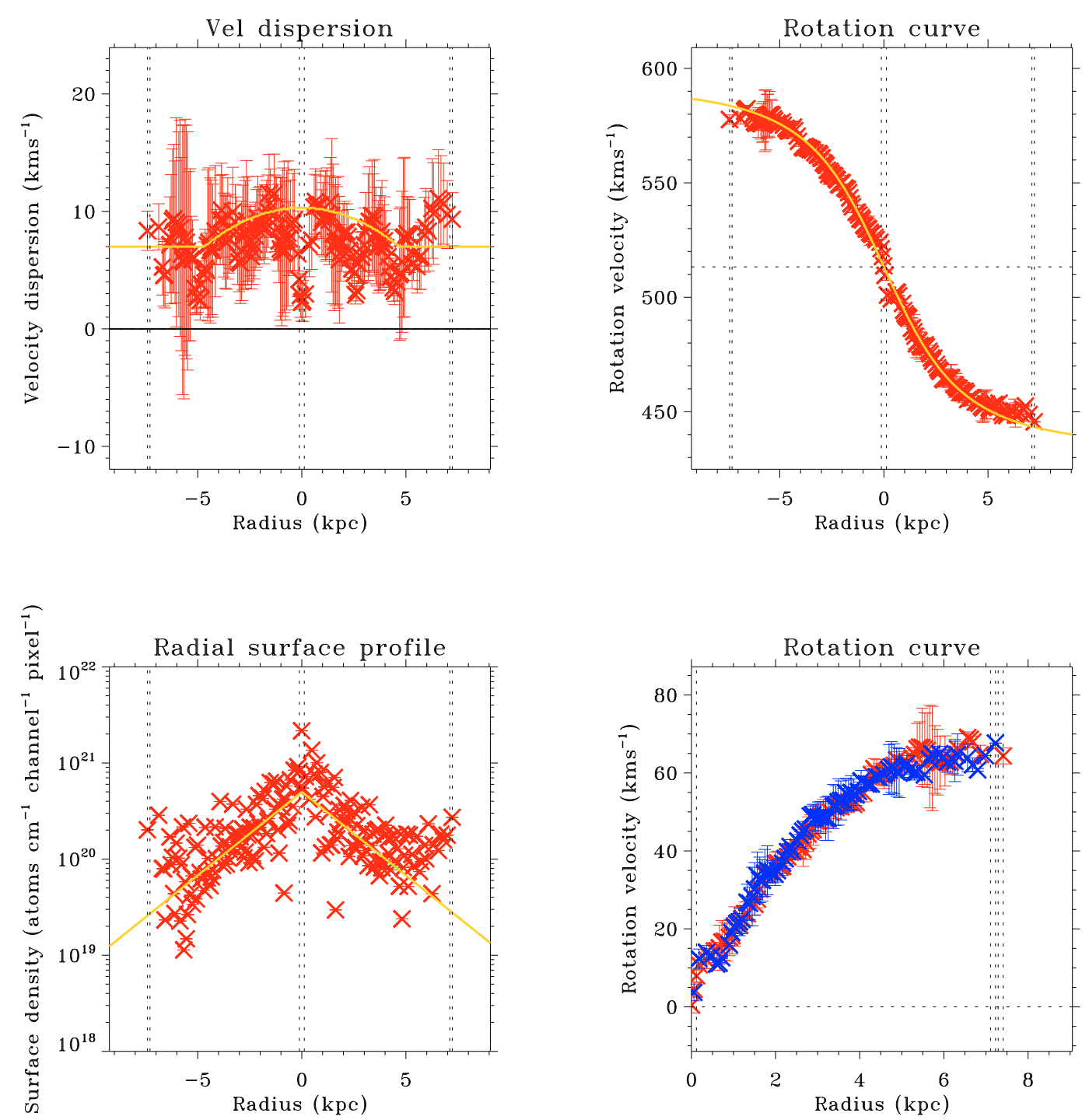

Fig. 4c. Fitted kinematics measured from an XV diagram observed with a telescope beam of $F W H M_{\theta}=300$ pc and to a peak signal-to-noise of 20. Beam-smearing correction is not necessary at this high resolution, as the scatter due to beam-smearing is much smaller than the scatter due to noise. This figure and the plots in Fig. $4 \mathrm{~b}$ demonstrate the effect of noise on the measured kinematics. To reduce the scatter we have averaged the measurements using a evenly weighted 7 pixel wide bin after fitting the whole XV diagram via the radial decomposition method. The dashed line are as in Fig. 4b.

- with the integrated flux of each Gaussian consisting of the flux at that radius.

Starting at the outermost radius on one side of the galactic centre, each line-of-sight is modelled as a sum of Gaussians, where each Gaussian simulates the gas at that radius. Due to the approximately exponential outer Hi distribution of most galaxies, the gas from radii near the line of nodes dominates the flux in each velocity profile slice through the XV diagram. At the outermost line-of-sight, the velocity profile is approximated as a single Gaussian as the flux can be approximated as coming from a single radial bin with $R^{\prime}=R_{\max }$. The properties $v(R), \sigma_{\mathrm{v}, \mathrm{HI}}(R)$, $\Sigma_{\mathrm{HI}}(R)$ at that radius can be directly measured at $R_{\max }$. In the next innermost radius, $R_{2}^{\prime}=R_{\max }-(1$ pixel $)$, the observed velocity profile is modelled as a superposition of the Gaussian with the known $R_{\max }$ kinematics, and the Gaussian with kinematics from the line-of-nodes at radius $R_{2}^{\prime}$ which is fitted for rotation, velocity dispersion and total flux. By proceeding inwards in this manner, the model never becomes ill-constrained as there are only 3 free parameters in the fit to any sightline.

The radial kinematics are thus obtained with radial sampling corresponding to the pixel size of the HI image cube along the galaxy major axis $R^{\prime}$. The derived kinematics from fitting to all slices of the raw XV diagram using in the above slice fit are shown in Fig. 2. The fit has been made to a synthetic galaxy with a constant velocity dispersion, a simple rotation curve and an exponential surface density distribution. The plot demonstrates that the radial decomposition method does an excellent job of recovering the actual galaxy structure and kinematics. Near the edge of the edge-on galaxy, the fits are systematically high in both the velocity dispersion and the surface density, because the model assumes that the outermost detected slice contains flux at a single radial bin. The overestimation of the velocity dispersion, causes the fitting process to compensate on the next few inner radii slice fits; thus the velocity dispersion fit oscillates around the actual value in the outer few slices. The small deviation in the centre 
Kinematic fit
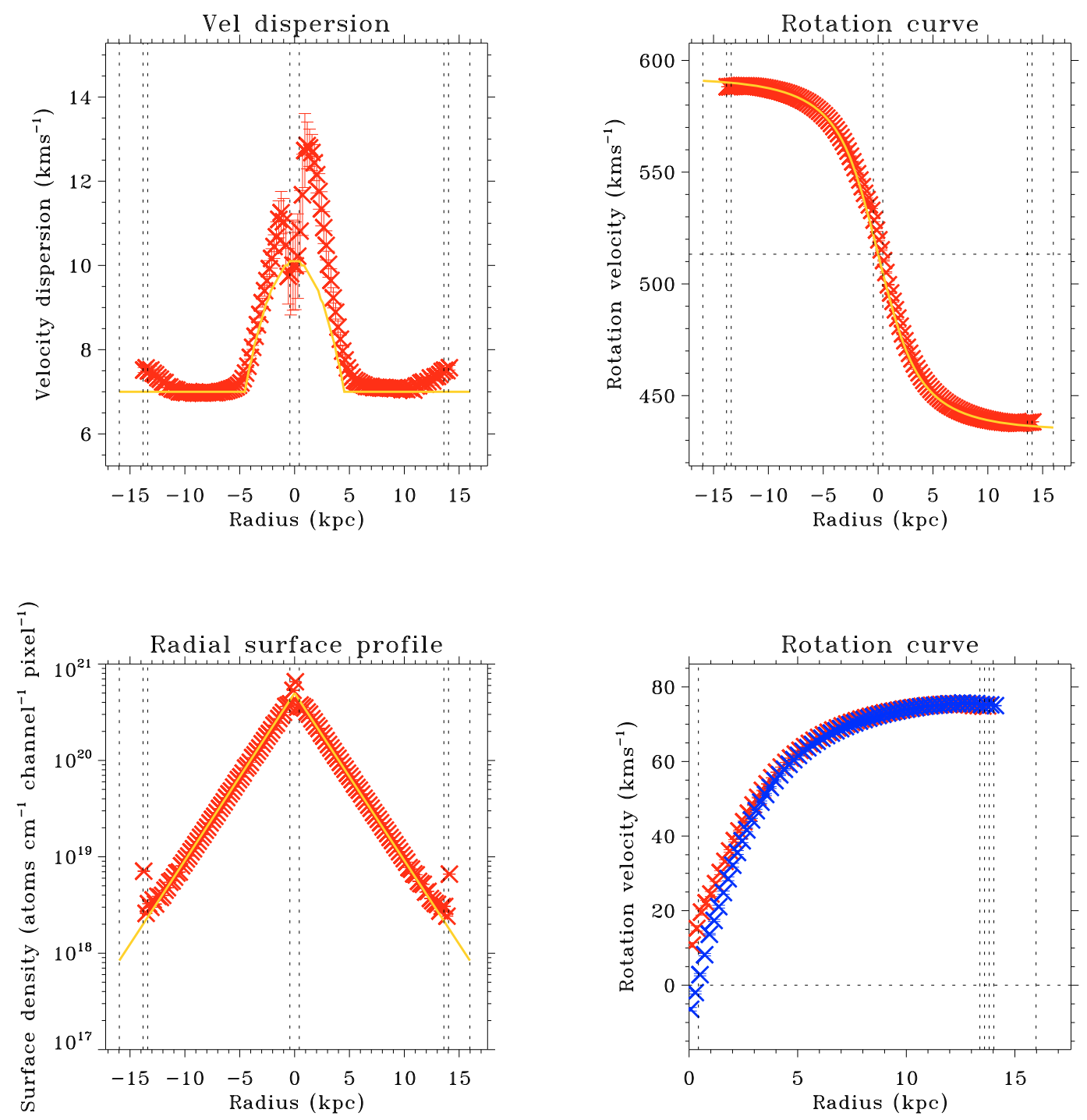

Fig. 5a. Fitted kinematics (without beam correction) measured from a noiseless XV diagram observed with a telescope beam of $F W H M_{\theta}=1 \mathrm{kpc}$. The plots shows that the rotation velocity exhibits a small beam-smearing effect and the velocity dispersion is broadened due to beam-smearing by $\sim 50 \%$ at the Galaxy centre. The dashed line are as in Fig. $4 \mathrm{~b}$.

slice is a numerical artifact. We remove this artifact by excluding the central fits within $0.5 \mathrm{kpc}$ of the galaxy centre. Although these plots show the derived fits to the XV diagram formed from a simple galaxy model with a constant velocity dispersion, simple surface density and rotation curve, the same fitting success is achieved for fits to synthetic galaxies with more complicated intrinsic kinematics.

\subsection{Instrumental broadening correction}

Fits of real galaxy observations also require correction for the instrumental broadening due to the signal response of the spectral channels of the interferometer correlator. For simplicity, the signal response function of the correlator channels is approximated as a Gaussian. Consequently, the dispersion due to both the HI velocity dispersion and the instrumental broadening is the quadratic sum of each property. Thus, the measured velocity dispersion is related to the intrinsic HI velocity dispersion and the dispersion $\sigma_{\mathrm{v}, \text { instr }}$ of a spectral channel with $F W H M_{\mathrm{v}, \text { instr }}=$ $2 \sigma_{\mathrm{v}, \text { instr }} \sqrt{2 \log 2}$ by:

$\sigma_{\mathrm{v}, \text { tot }}=\sqrt{\sigma_{\mathrm{v}, \mathrm{HI}}^{2}+\sigma_{\mathrm{v}, \text { instr }}^{2}}$

This allows the intrinsic HI velocity dispersion to be easily derived from each Gaussian fit.

\subsection{Beam correction}

Any method that aims to recover spatial resolution on scales of the image pixels must also correct for the convolution effect of the telescope synthesised beam. The beam is approximated an elliptical Gaussian, which is near circular for objects at an appropriate declination relative to the latitude of the observatory. The telescope beam causes a smearing effect along the spatial axes of the HI channel map cube by convolving the flux distribution with a 2D Gaussian. As the XV map is formed by integrating 
Kinematic fit
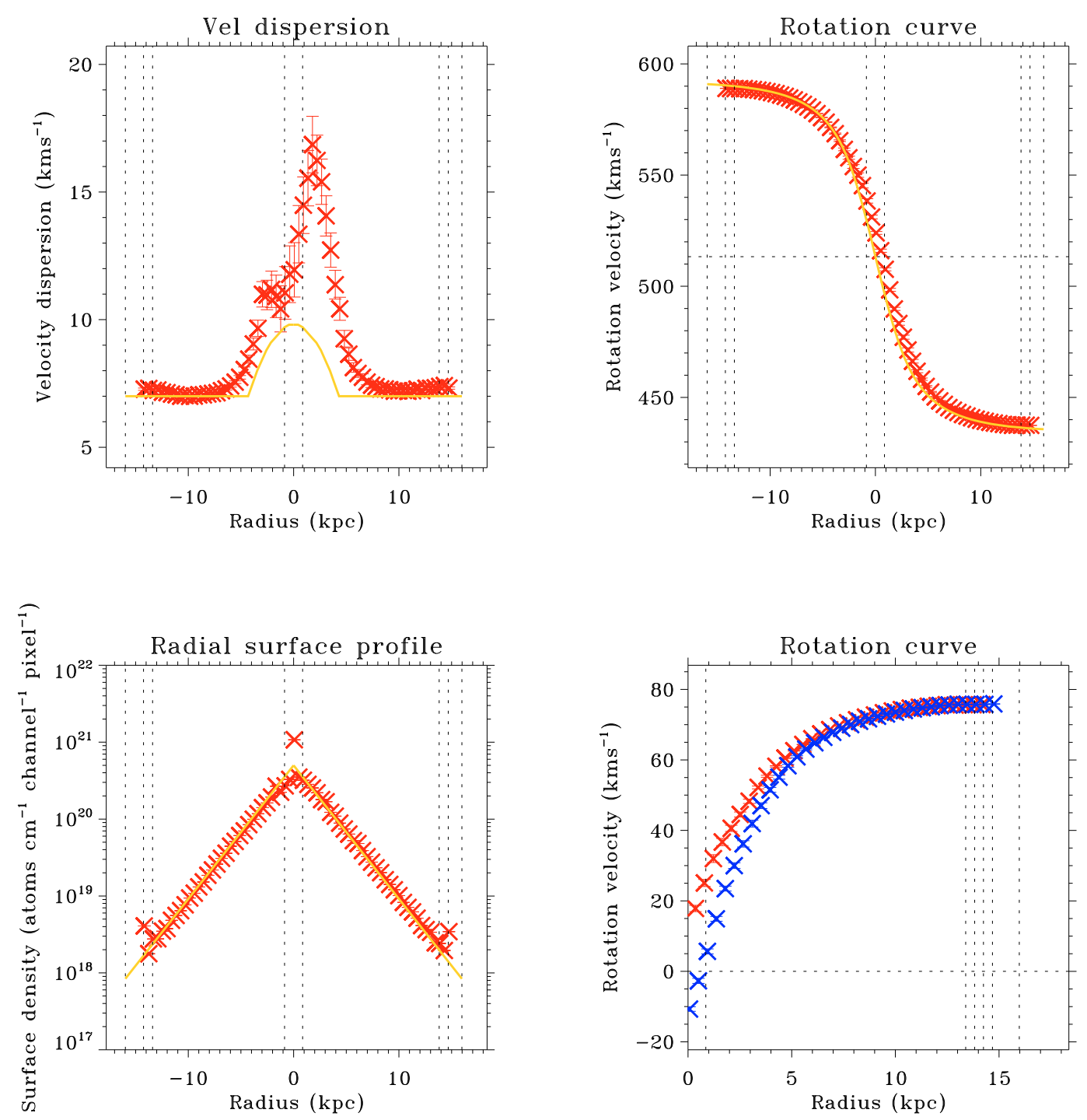

Fig. 5b. Fitted kinematics (without beam correction) measured from a noiseless XV diagram observed with a telescope beam of $F W H M_{\theta}=2.0 \mathrm{kpc}$. The plots shows that beam-smearing by a $2 \mathrm{kpc}$ beam causes a large error in the inner rotation velocity and a $\sim 70 \%$ increase of the measured central velocity dispersion. The dashed line are as in Fig. $4 \mathrm{~b}$.

over $z$, then providing the beam is near circular, the beam effectively smears the spatial axis of the XV map by a 1D Gaussian with FWHM equal to that of the beam, along the $R^{\prime}$ axis.

Beam smearing is a problem when the galaxy kinematics vary strongly on intervals along the major axis smaller than the $F W H M$ of the synthesised beam. In practise, the largest variations usually occur in the inner region where the rotation curve rises steeply and to a lesser extent in any region with variable kinematics due to heating processes in the ISM.

In the following Sect. 3.3, we describe in detail how we incorporate an iterative process to check the derived kinematics measured from the observed XV map. Briefly the process works as follows. Firstly, the first-pass kinematics from the the kinematic model is used to build a synthetic galaxy XV diagram using Eq. (6), which is then convolved by the known telescope resolution to simulate the actual observational effects. This simulated XV diagram is then fitted with the radial decomposition method and the resulting kinematics compared to the original fitted kinematics. Where the difference between the kinematic fits is greater than a critical level the kinematic models are incremented and the process repeated until the fitted kinematics and the simulated galaxy resemble the original fitted kinematics and the observed galaxy distribution.

Initially beam correction was attempted by iterating the fitting process as described above. However the velocity dispersion fits are particularly sensitive to noise, and it was found to be difficult to correct the velocity dispersion by iterative fitting if the first pass fits were too deviant from the true fit, as often occurs when fitting galaxies with steep inner rotation curves that have been observed with a large beam. The optimal method was found to be using the beam correction method applied by Kregel \& van der Kruit (2004) whereby instead of fitting the actual observed slice, the slice is first corrected by subtracting the flux contamination due to beam-smearing of flux in nearby slices. The residual profile is then fitted using the radial decomposition method. As the radial decomposition method fits progressively inwards, the kinematics are known at radii larger than the slice of interest $R^{\prime}$, thus it is possible to form a raw "mini-XV diagram" comprising solely the slices at higher $R^{\prime}$. However to correctly derive the flux contamination due to beam smearing a raw 
Kinematic fit
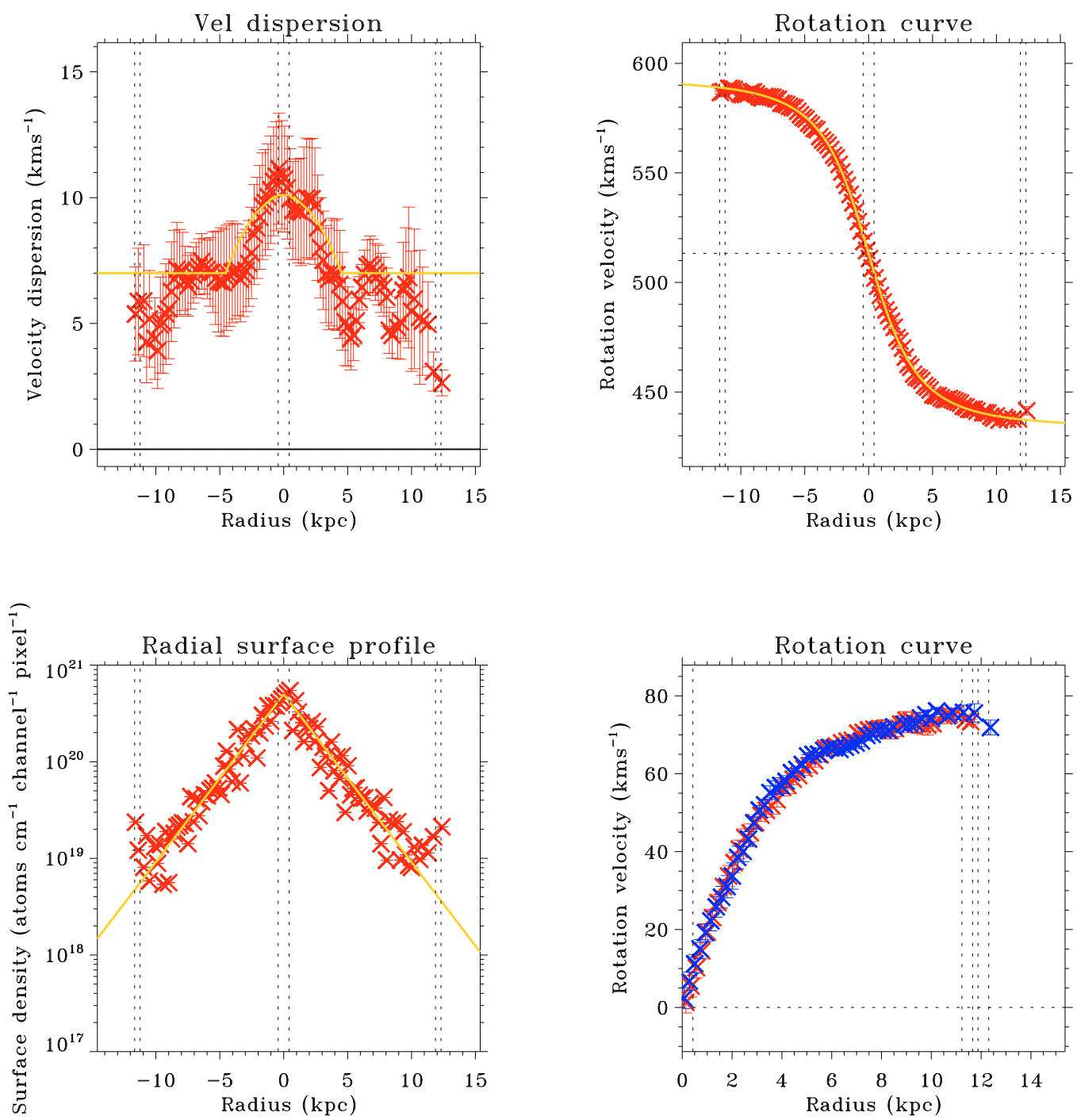

Fig. 6a. Fitted kinematics (with beam correction) measured from XV diagram observed with a telescope beam of $F W H M_{\theta}=1 \mathrm{kpc}$ and with a peak signal-to-noise of 80 . To reduce the scatter in the measured kinematics, the velocity dispersion and rotation curve were averaged (post fitting) over an evenly weighted bin of width of 5 pixels. In Fig. 6b, the XV diagram built with the best-fit measured kinematics and observed to the same telescope specifications is compared to the original XV diagram.

"mini-XV diagram" must be built spanning sufficient slices on both sides, but excluding the slice of interest. This mini-XV diagram is convolved along $R^{\prime}$ by a Gaussian of $F W H M$ equal to that of the beam $F W H M_{\theta}$ along $R^{\prime}$, to determine the beamsmearing component of the flux in the slice of interest.

Consider the observed beam-smeared velocity profile at the slice of interest $F_{0}$. This velocity profile is the sum of the intrinsic profile $f_{0}$ weighted by the beam centre $b_{0}$ and the flux of all nearby slices weighted by their respective beam weights $b_{n}$, where $b_{n}$ becomes negligible for slices further than a few $F W H M_{\theta}$ 's from the slice of interest. Thus

$F_{0}=f_{0} b_{0}+\sum_{n=1}^{\infty} f_{n} b_{n}+\sum_{n=-1}^{-\infty} f_{n} b_{n}$.

Here each beam weight $b_{n}$ is the flux of a normalized Gaussian in the $n$th pixel from the beam centre. Therefore, the intrinsic velocity profile is

$f_{0}=\frac{F_{0}-\left(\sum_{n=1}^{\infty} f_{n} b_{n}+\sum_{n=-1}^{-\infty} f_{n} b_{n}\right)}{b_{0}}$, where $b_{0}$ is the peak flux of a normalised Gaussian beam

$$
b_{0}=\frac{1}{\sqrt{2 \pi} \sigma_{\theta}} \text { with } \sigma_{\theta}=\frac{F W H M_{\theta}}{2 \sqrt{2 \log 2}} \text {. }
$$

Beam correction is thus undertaken by determining $\sum_{n=1}^{\infty} f_{n} b_{n}$ and $\sum_{n=-1}^{-\infty} f_{n} b_{n}$ by convolving a raw mini-XV diagram formed from all slices but the slice of interest. Then this beam smearing component is subtracted from the observed slice, and the normalisation $b_{0}$ is applied. The raw un-beam-smeared mini-XV diagram is built using Eq. (6). This requires the intrinsic kinematics which are only known for radii at $R>R^{\prime}$ determined from fits outside the slice of interest. To estimate both parts of the mini-XV diagram, we conduct an initial radial decomposition fit across the whole observed XV diagram. This first loop fit is accurate, except near the galaxy centre, where the high gradients of rotation and velocity dispersion over $R^{\prime}$ distort the line profiles. The kinematics near the galaxy centre are estimated (for beam smearing correction) by extrapolating inwards using the higher quality fits over the rest of the galaxy. Subsequent iterations of 

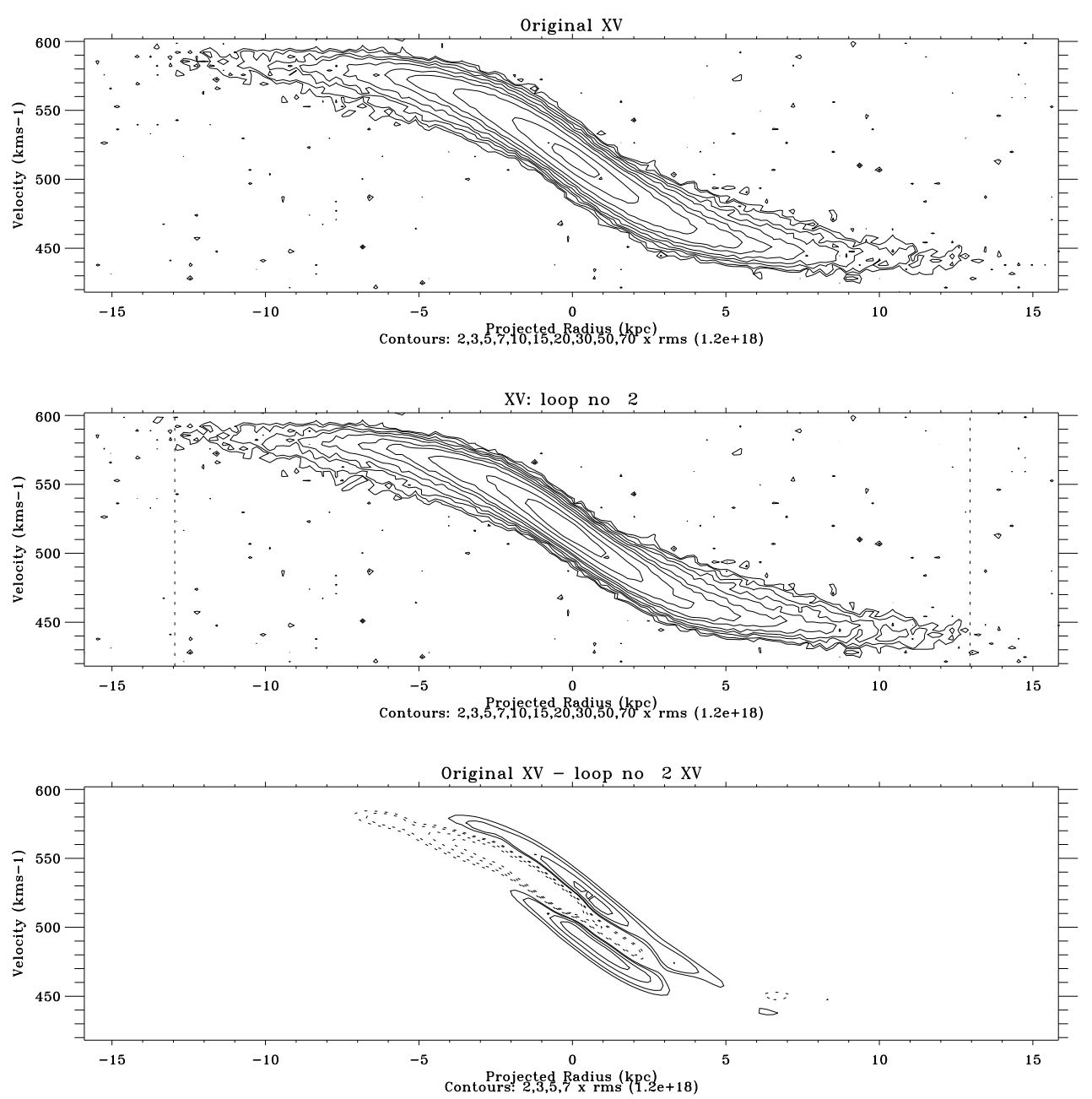

Fig. 6b. Top: original XV diagram observed with a $F W H M_{\theta}=1 \mathrm{kpc}$ telescope beam and peak $S / N$ of 80 . Middle: the XV diagram built using the best-fit measured kinematics and observed with the same telescope resolution and sensitivity limits. The measured kinematics used to build the middle plot are shown in Fig. 6a. Bottom: the difference between the top and middle diagrams. Contour units are 2, 3, 5, 7, 10, 15, 20, 30, 50, 70 times the rms noise in the channel maps.

the radial decomposition fitting process can then be beam corrected using the mini-XV diagram method, whereby the mini$\mathrm{XV}$ diagram is formed using these estimated kinematics.

This beam correction is essentially a deconvolution of the XV map to remove the effect of the telescope beam. As such the sampling of the beam, i.e. the number of pixels per beam, affects the success of the deconvolution, as the deconvolution process amplifies the noise. The recommended sampling for deconvolution is 3 pixels per beam $F W H M_{\theta}$. To recover a slightly higher spatial resolution, which is useful when modelling the more distant galaxies, we used a beam sampling of 5 pixels when forming the HI image cubes. Both sampling densities work well, however a yet higher sampling density $(\gtrsim 8)$ dramatically reduces the peak signal-to-noise of the beam corrected XV slice, as the telescope beam naturally limits the spatial resolution of the observations. As the galaxies in our sample lie at recession velocities from 400-2500 $\mathrm{km} \mathrm{s}^{-1}$, and distances of 3-30 Mpc, the $F W H M_{\theta}$ beam size of the galaxy observations is $0.1-1.0 \mathrm{kpc}$. Given that the kinematics can be recovered on a scale of $1 / 5$ of the beam $F W H M_{\theta}$, this leads to a recovered spatial resolution of 20-200 pc for our galaxies.

The beam correction is necessary for galaxies with large beam sizes $\gtrsim 500 \mathrm{pc}$, as for these galaxies the beam smearing is sufficient to distort the slice shape away from a sum of
Gaussians, over the major axis range where the kinematics vary significantly over the span of the beam. Without beam correction, radial decomposition fitting of the inner disk will tend to oscillate with a large scatter, due to the intrinsicly non-multiGaussian shape of the velocity profiles in these sightlines. This occurs as an erroneous fit to gas on the line of nodes in any one sightline will cause the fit to the next inwards radius to overcompensate, resulting in an oscillating fit over the beam-smeared domain.

\subsection{Iterating to ensure self-consistent fits}

To assess and improve the quality of the fitted kinematics, the process is iterated by building a model galaxy from the original fitted kinematics, simulating the observations and comparing the fitted properties of the model $f_{n}\left[v(R), \sigma_{v, \mathrm{HI}}(R), \Sigma_{\mathrm{HI}}(R)\right]$ galaxy XV diagram to the original fitted kinematics $f_{1}\left[v(R), \sigma_{v, \mathrm{HI}}(R), \Sigma_{\mathrm{HI}}(R)\right]$. A schematic showing

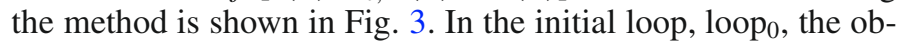
served XV map is fitted to obtain a rough kinematic model that is used to perform beam correction. This mini-XV diagram is then used in the next fit to the observed XV map, which we will call loop l $_{1}$ The radial functions of HI surface density, rotation and velocity dispersion fitted in $\operatorname{loop}_{1}$ fit, which are the 
Kinematic fit
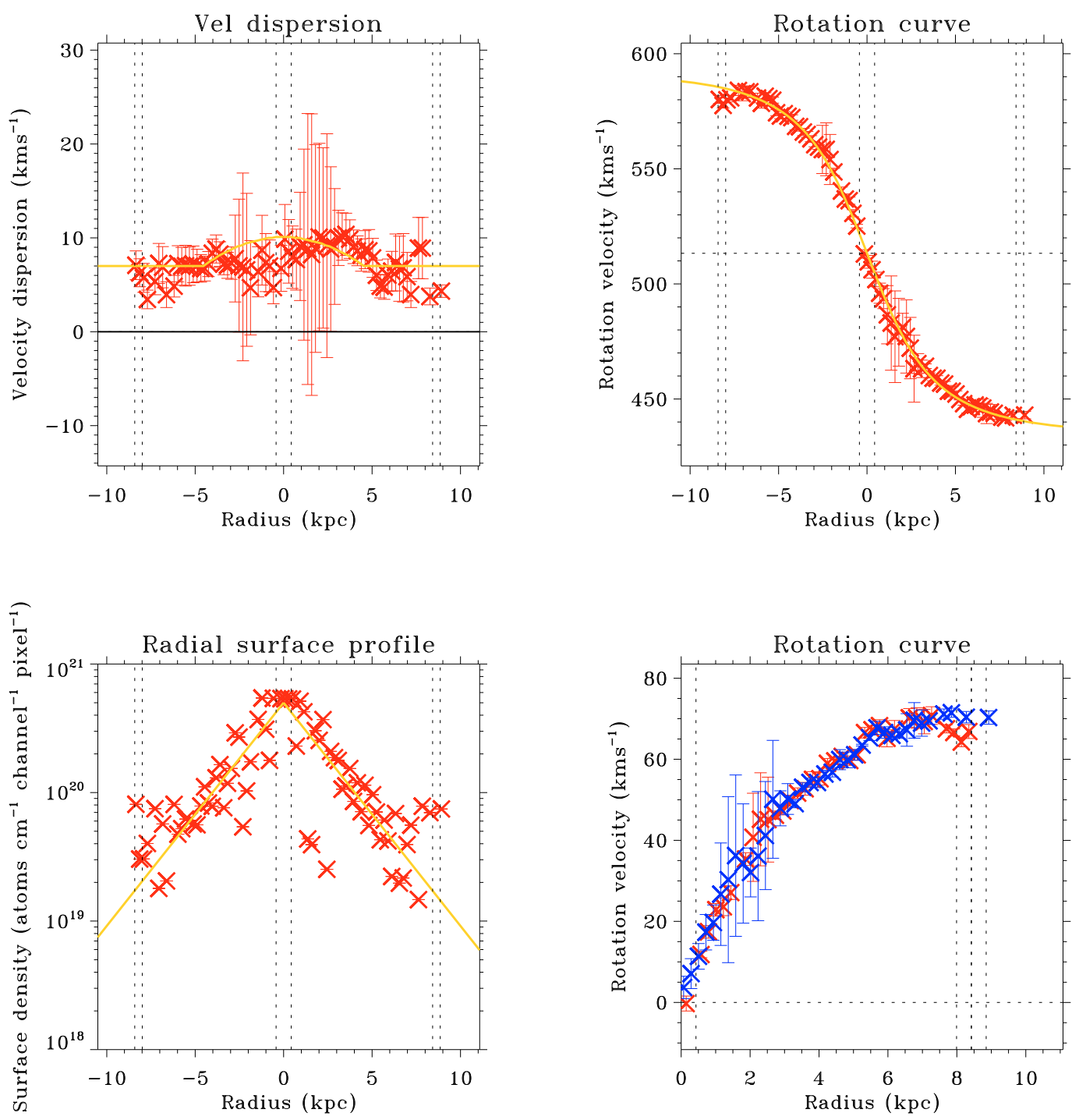

Fig. 7a. Fitted kinematics (with beam correction) measured from XV diagram observed with a telescope beam of $F W H M_{\theta}=1 \mathrm{kpc}$ and with a peak signal-to-noise of 30 . Although the rotation velocity and the surface density are well recovered, the plot of the measured HI velocity dispersion shows that a peak signal-to-noise of 30 is not high enough to perform beam-smearing correction for this kinematic model and moderate spatial resolution. The measurements are binned over 3 pixels post-fitting, however averaging only improves the measurement when it is accurate. By comparison, the observation with a larger beam to the same signal-to-noise recovers more of the HI emission distribution and thus recovers the radial shape of the HI velocity dispersion (compare to Fig. 9a). In Fig. 7b, the XV diagram built with the best-fit measured kinematics and observed to the same telescope specifications is compared to the original $\mathrm{XV}$ diagram.

first fits incorporating beam correction, are then used as the first kinematic model for a new XV diagram. By iterating the fitting process, and incrementing the kinematic model between fits, the process is repeated until the fitted kinematics from the model equal the original fitted kinematics with beam correction to within a critical threshold.

By intrinsically incorporating fitting of the gas velocity dispersion into the radial decomposition method, it is possible to replicate the observed XV diagram from the best fit kinematics as all three functions characterising the XV map are obtained: rotation curve $v(R)$, HI velocity dispersion $\sigma_{v, \mathrm{HI}}(R)$ and the deprojected HI surface density $\Sigma_{\mathrm{HI}}(R)$. Unlike earlier methods of measuring rotation curves or HI velocity dispersion, this method is internally self-consistent.

\section{Quality testing on synthetic galaxy observations}

The quality of the derived kinematics is quantified in two ways. Firstly, we calculate the total absolute difference between the best fit and original fit for each of the three functions: $v(R)$, $\sigma_{v, \mathrm{HI}}(R)$ and $\Sigma_{\mathrm{HI}}(R)$. This difference is summed over all measured slices across the galaxy and divided by the number of measured slices to present a average difference at each radius.

Secondly, the XV map formed from the best fit kinematics is compared to the observed $\mathrm{XV}$ map by computing the residual. The residual map (with absolute values for the residuals) is normalised by the observed XV map to obtain a total percentage difference between the actual galaxy XV diagram and the best fit model. The residual XV map is a good indicator of surface 

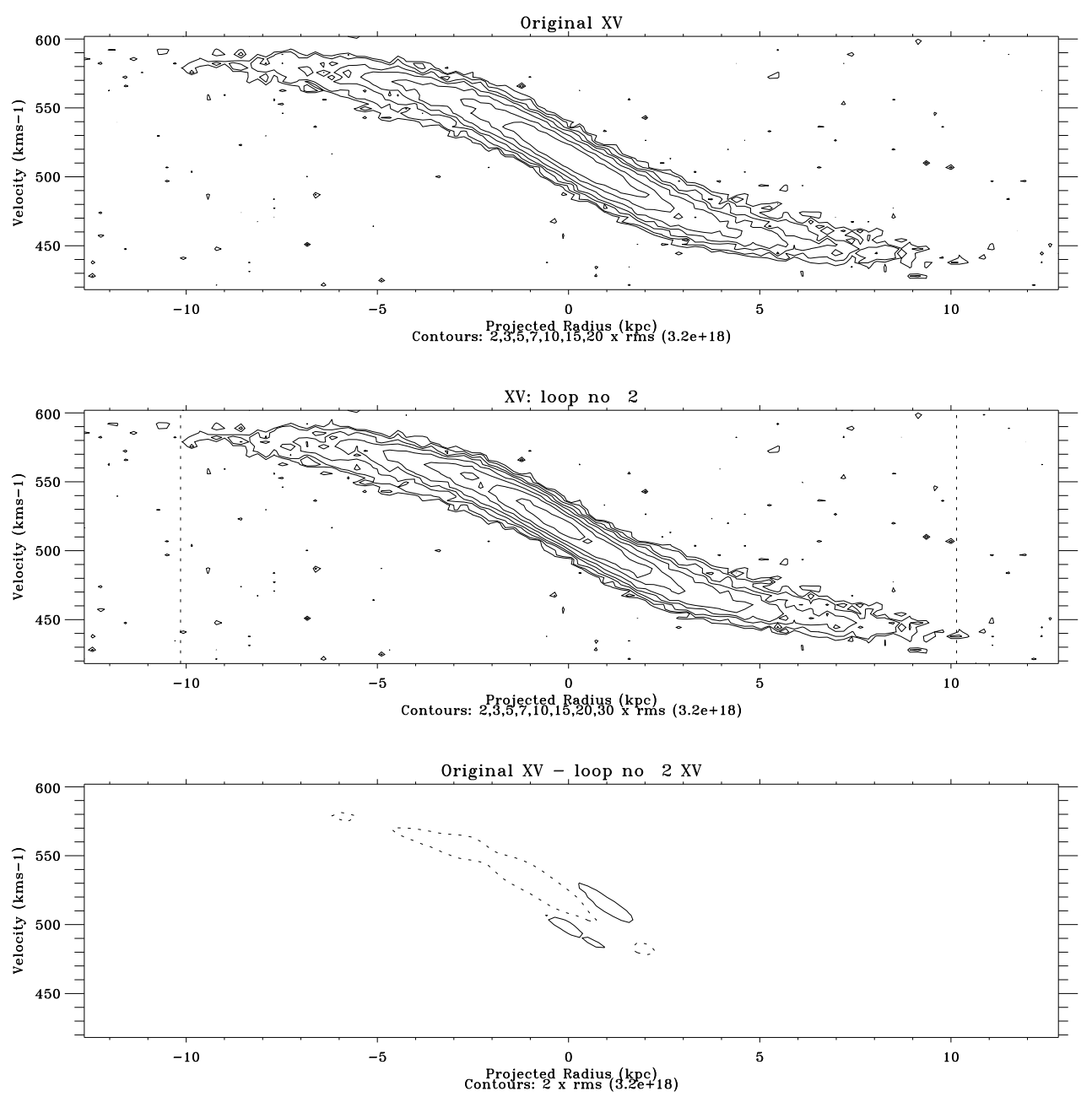

Fig. 7b. Top: original XV diagram observed with a $F W H M_{\theta}=1 \mathrm{kpc}$ telescope beam and peak $S / N$ of 30 . Middle: the XV diagram built using the best-fit measured kinematics and observed with the same telescope resolution and sensitivity limits. The measured kinematics used to build the middle plot are shown in Fig. 7a. Bottom: the difference between the top and middle diagrams. Contour units are 2, 3, 5, 7, 10, 15, 20, 30 times the rms noise in the channel maps.

density and rotation curve accuracy, but is relatively insensitive to errors in the measured velocity dispersion.

\subsection{Effect of noise}

The noise level is of most concern when fitting observations that suffer significant beam-smearing, as the beam correction method uses deconvolution, which is particularly sensitive to noise. To isolate the effect of noise from that due to beamsmearing, we tested the radial decomposition method on high spatial resolution XV maps (observed with $F W H M_{\theta}=300 \mathrm{pc}$ ) with different sensitivities corresponding to peak signal-to-noise ratios ranging from 20 to 80 . In each case the same galaxy model is used, featuring a differential rotation curve, an exponential face-on HI surface density (with a HI scalelength of $2.5 \mathrm{kpc}$ ) and a flat outer HI velocity dispersion that rises parabolicly in the inner disk from an outer plateau of $7 \mathrm{~km} \mathrm{~s}^{-1}$ to a central peak of $10 \mathrm{~km} \mathrm{~s}^{-1}$. In Fig. 4a we show the XV diagrams for this synthetic galaxy without noise and with noise introduced at peak $S / N$ levels of 80 and 20. The upper diagrams, labelled "raw unedged", are the pure synthetic diagrams that would be observed in the absence of noise. The lower ones show the effects of introducing noise. The radial (horizontal) scales in the diagrams left and right are different and have been chosen such that it the observable radial extents fit within the frames. This shows the severe effects of noise.

In Figs. 4b and 4c, we show the measured kinematics from the simulated observation of this model galaxy with noise levels corresponding to peak signal-to-noise ratios of 80 and 20. In each figure the actual kinematics are shown in grey (yellow). As mentioned above, both the rotation curve and the radial surface density are well measured, even in the low sensitivity $S / N=20$ and $S / N=30$ figures. However, the HI velocity dispersion is particularly sensitive to high noise levels that submerge the outer HI distribution. Comparison of an ideal XV map and XV map generated with finite telescope sensitivity and resolution (see Fig. 4a) demonstrates the limited HI detected at low sensitivities. But despite the low flux recovery, these simulations show that low sensitivity observations still yield reasonably accurate kinematics, albeit with dramatically increasing measurement error at lower sensitivities.

To reduce the scatter, an evenly weighted running mean was applied to the measured fits after the XV diagram was fitted with the radial decomposition method. By averaging the measured kinematics every 3 or more pixels in radius, it was possible to reduce the scatter in measured velocity dispersion and recover the 
Kinematic fit
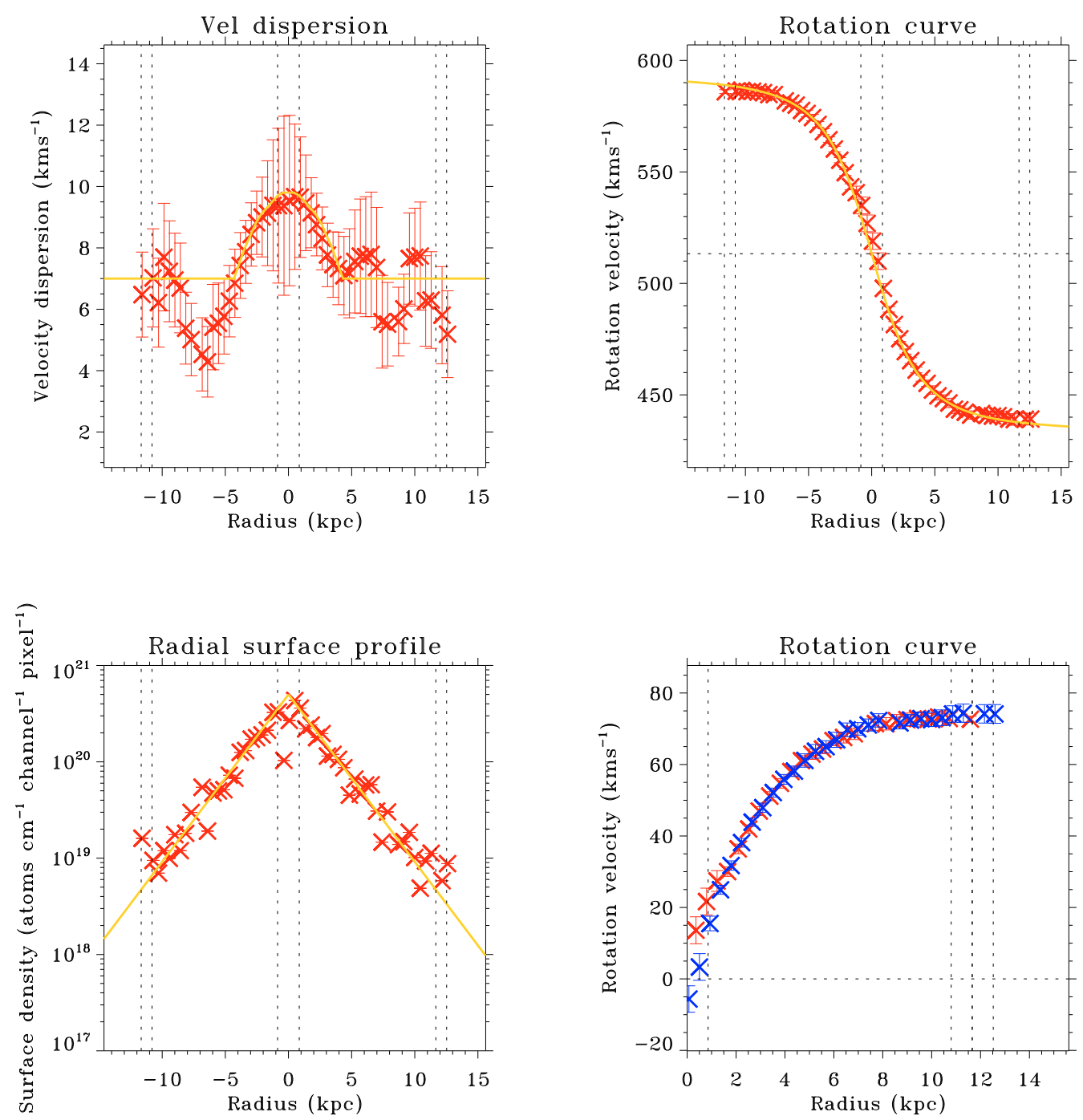

Fig. 8a. Fitted kinematics (with beam correction) measured from XV diagram observed with a telescope beam of $F W H M_{\theta}=2 \mathrm{kpc}$ and with a peak signal-to-noise of 80 . To reduce the scatter in the measured kinematics, the velocity dispersion and rotation curve were averaged (post fitting) over an evenly weighted bin of width of 3 pixels. In Fig. 8b, the XV diagram built with the best-fit measured kinematics and observed to the same telescope specifications is compared to the original XV diagram.

radial trend of the kinematics with good confidence. This proved to be more revealing, although it effectively lowered the spatial resolutions of the measurement. The simulations show that measurements of galaxies observed with peak $S / N<100$ benefit from this post-fit averaging. Given an XV image gridded with a beam sampling density of 5 (i.e. each pixel is $1 / 5$ of the beam $F W H M_{\theta}$ ) and beamwidth $F W H M_{\theta}=300$ pc, the final postaveraging resolution is still high at 180 or $300 \mathrm{pc}$ after averaging over a 3-pixel or 5-pixel wide bin. A post fit averaging interval of 5 was used for the $S / N_{\max }=80$ dataset; while this was increased to 7 for the noisy $S / N_{\max }=20 \mathrm{XV}$ map. As shown by the plots in Figs. $4 \mathrm{~b}$ and $4 \mathrm{c}$, it was possible to recover the radial trend in the velocity dispersion for $\mathrm{XV}$ diagrams with these kinematics, providing the peak $S / N>20$.

In Figs. $4 b$ and $4 c$, we show the measured kinematics from the simulated observation of this model galaxy with noise levels corresponding to peak signal-to-noise ratios of 80 and 20 . In each figure the actual kinematics are shown in grey (yellow). As mentioned above, both the rotation curve and the radial surface density are well measured, even in the low sensitivity
$S / N=20$ and $S / N=30$ figures. However, the HI velocity dispersion is particularly sensitive to high noise levels that submerge the outer HI distribution. Comparison of an ideal XV map and $\mathrm{XV}$ map generated with finite telescope sensitivity and resolution (see Fig. 4a) demonstrates the limited HI detected at low sensitivities. But despite the low flux recovery, these simulations show that low sensitivity observations still yield reasonably accurate kinematics, albeit with dramatically increasing measurement error at lower sensitivities.

In the slice fits of the edge of the galaxy, the measurements are biased by two effects. Firstly, in the outer few slices the radial decomposition method models all the flux as originating from a single radial bin. Consequently, the measured velocity dispersion is broadened by the projected rotation of gas away from the line-of-nodes, i.e. at radii higher than $R=R^{\prime}$; similarly the measured surface density is overestimated due to the attribution of flux away from the lines-of-nodes. This problem is systematic of the radial decomposition method, although it is relatively unimportant as it only effects the outer few slices. 

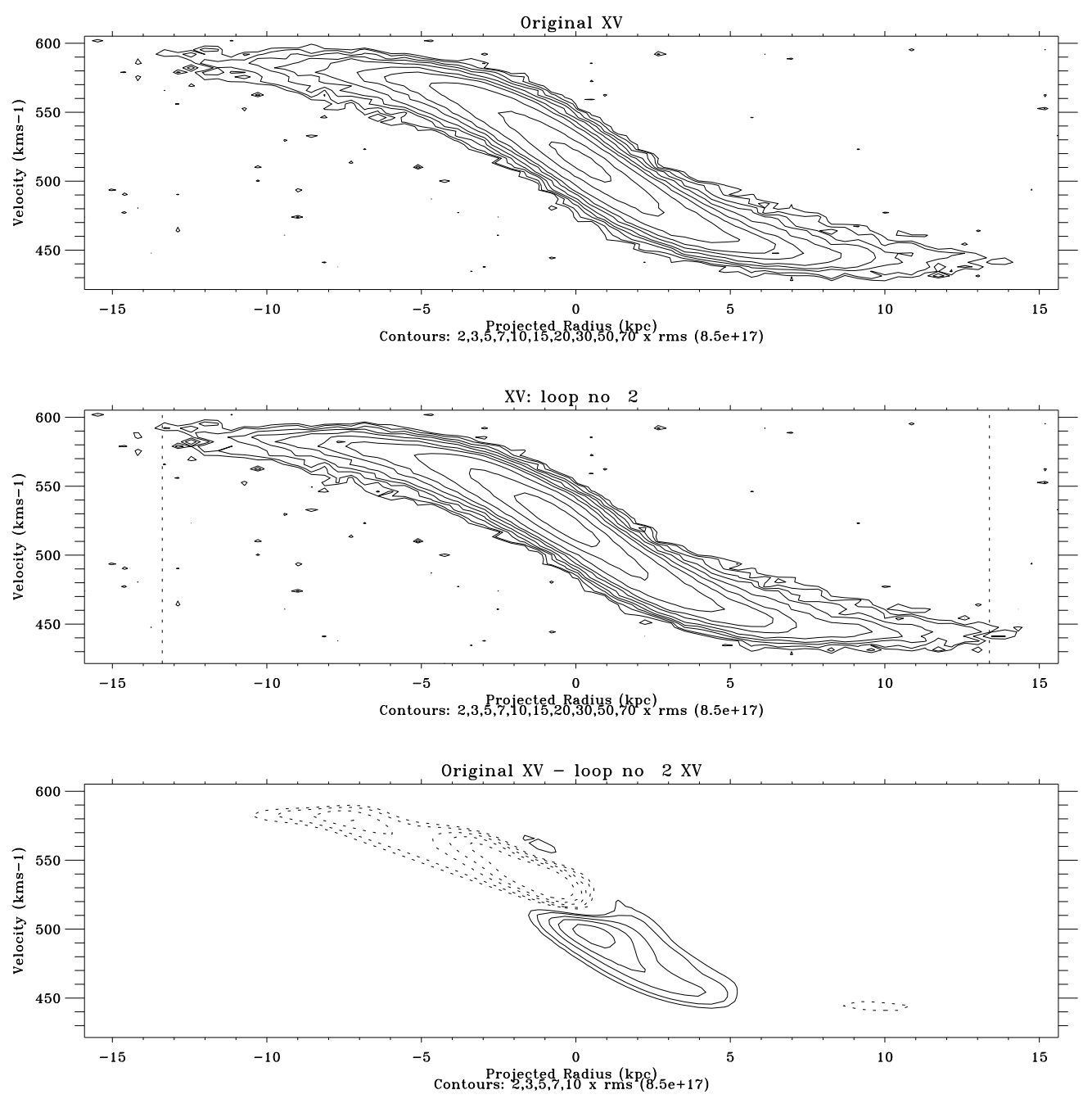

Fig. 8b. Top: original XV diagram observed with a $F W H M_{\theta}=2 \mathrm{kpc}$ telescope beam and peak $S / N$ of 80 . Middle: the XV diagram built using the best-fit measured kinematics and observed with the same telescope resolution and sensitivity limits. The measured kinematics used to build the model XV plot are shown in Fig. 8a. Bottom: the difference between the top and middle diagrams. Contour units are 2, 3, 5, 7, 10, 15, 20, 30, 50, 70 times the rms noise in the channel maps.

Secondly, the fits to the velocity profile of slices with very low peak $S / N(\$ 5-8)$ are biased by noise peaks dominating the underlying flux profile. The radial decomposition fitting algorithm starts fitting where it finds velocity profile slices with flux greater than $2 \sigma$ in 4 adjacent velocity channels. A galaxy with an exponentially declining radial surface density typically has a peak $S / N \lessgtr 5-8$ in the slices at the outermost $1-2 \mathrm{kpc}$. Gaussian fitting of these low $S / N$ velocity profiles is particularly sensitive to underestimating the velocity dispersion, due to fitting the noise peaks instead of the underlying flux profile. As noise peaks typically have a $F W H M$ of $1-2$ velocity channels, the velocity dispersion measurements in these outer few kpc is contaminated with noise fits exhibiting a velocity dispersion of $\sigma_{v} \sim(1-2$ channels $) /(2 \sqrt{2 \log 2}) \sim 1-2 \mathrm{~km} \mathrm{~s}^{-1}$. As the radial decomposition fitting method progressively fits inwards using the outer fits to model the gas at radii away from the line-ofnodes in each velocity profile, erroneous fits cause subsequent measurements to oscillate around the correct value. These erroneous fits only effect the adjacent few slice fits as the typically near-exponential radial surface density in the outer HI disk causes the flux contribution of outer radii to be relatively small.

In summary, the radial decomposition method is able to successfully measure the radial distributions of the velocity dispersion, rotation velocity and surface density for XV diagram observations with peak $S / N$ greater than 20 . This could perhaps be undertaken with lower $S / N$ maps, if the observation is actually detecting the bulk of the actual HI distribution, as could be the case on a galaxy with an intrinsically shallow radial surface density. The method breaks down for lower $S / N$ maps which are dominated by the noise over a larger major axis extent of the detected gas distribution. Also, as can be seen from the above figures, the major limitation of the low signal-to-noise observation of the above galaxy model is that one is only detecting the inner half of the gas disk, because the outer disk HI lies below the $2 \sigma$ noise level. The outermost radius with sufficient flux above $2 \sigma$ to allow kinematic fitting is marked by vertical dashed lines on the XV maps observed with different noise limits in Fig. 4a.

\subsection{Effect of spectral resolution}

Modern radio telescope array correlators of the ATCA and VLA have a spectral resolution of 3-5 $\mathrm{km} \mathrm{s}^{-1}$, when configured for a frequency bandwidth sufficient to cover the rotational velocity range of a small-to-medium spiral galaxy $\left(v_{\max } \lesssim 200 \mathrm{~km} \mathrm{~s}^{-1}\right)$. This is sufficient to resolve the $F W H M$ due to the typical intrinsic 
Kinematic fit
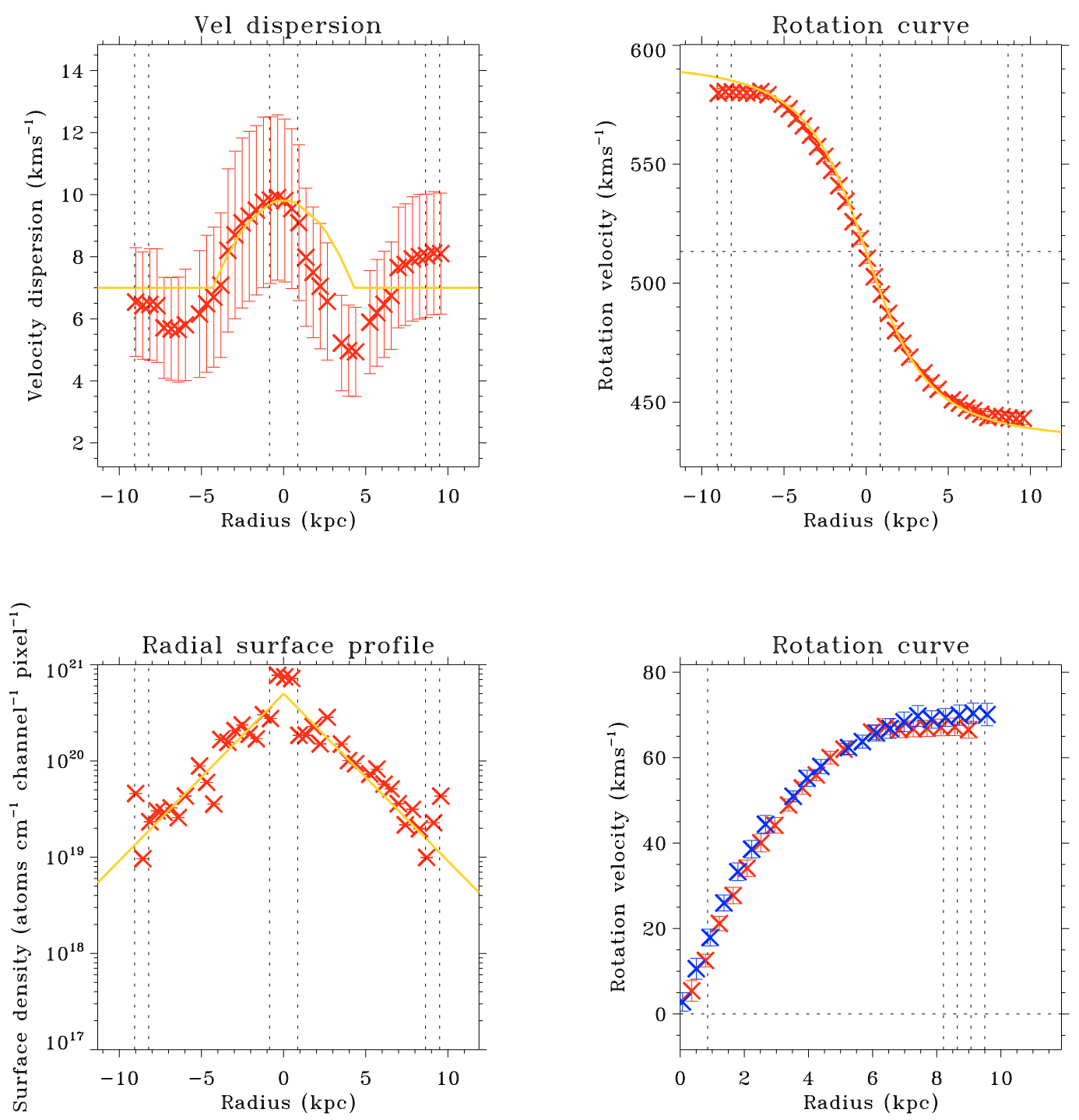

Fig. 9a. Fitted kinematics (with beam correction) measured from XV diagram observed with a telescope beam of $F W H M_{\theta}=2 \mathrm{kpc}$ and with a peak signal-to-noise of 30 . To reduce the scatter in the measured kinematics, the velocity dispersion and rotation curve were averaged (post fitting) over an evenly weighted bin of width of 9 pixels. In Fig. 9b, the XV diagram built with the best-fit measured kinematics and observed to the same telescope specifications is compared to the original XV diagram. The plots show that the larger beam of this observation allows the shape of the velocity dispersion to be recovered more accurately than a higher resolution observation with a $1.0 \mathrm{kpc}$ telescope beam, observed to the same noise limit (compare to Fig. 7a).

gas velocity dispersion of $7 \mathrm{~km} \mathrm{~s}^{-1}$ (the commonly adopted value) by 3-5 channels. For this reason we did not investigate how spectral resolution changes affected the measurement accuracy, although it should probably be considered if low HI velocity dispersions are found. All measurements of simulated HI observations were corrected for instrument broadening, according to Eq. (9).

\subsection{Effect of spatial resolution}

The shape of the beam is the largest factor apart from the sensitivity affecting the accuracy of the measured kinematics. But the effect of the beam smearing on the measured HI kinematics is only serious if the intrinsic galaxy kinematics has a steep gradient over the scale of the beam. As a result, the observed
HI distribution is more distorted over projected radial domains with steep gradients of rotation, velocity dispersion or surface density than those parts of the galaxy with shallower gradients. In practice, the observed HI distribution of a small galaxy with a maximum rotation speed of $\lesssim 100 \mathrm{~km} \mathrm{~s}^{-1}$ and a slowly rising inner velocity dispersion is only minimally distorted by a telescope beam with $F W H M_{\theta}=300$ pc. (See Figs. 5a and b to view the magnitude of the beam-smearing effects of different the beam size on the measured kinematics of the model galaxy.)

Calculation of the dark halo mass density requires smooth functions of the kinematics. Consequently, it is more important to determine the gas kinematics on scales of $1 \mathrm{kpc}$, than $100 \mathrm{pc}$. The sensitivity of the observations and accuracy of the radial kinematics was improved by convolving the observations by a larger beam size prior to fitting. This is demonstrated in Figs. $7 \mathrm{a}$ and $9 \mathrm{a}$ which show the measured kinematics using beam 

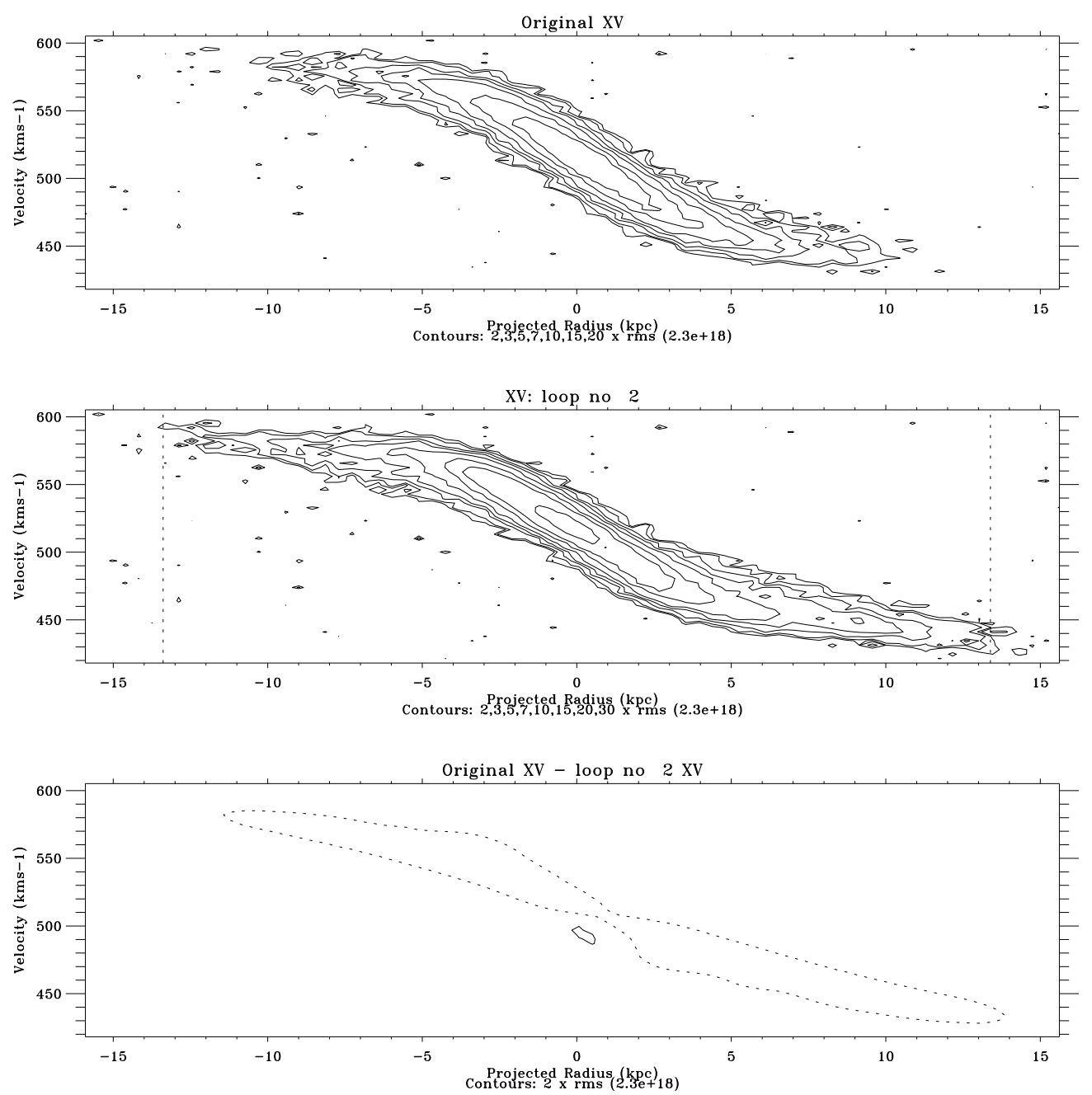

Fig. 9b. Top: original XV diagram observed with a $F W H M_{\theta}=2 \mathrm{kpc}$ telescope beam and peak $S / N$ of 30 . Bottom: the XV diagram built using the best-fit measured kinematics and observed with the same telescope resolution and sensitivity limits. The measured kinematics used to build the bottom plot are shown in Fig. 9a. Bottom: the difference between the top and middle diagrams. Contour units are 2, 3, 5, 7, 10, 15, 20, 30 times the rms noise in the channel maps.

correction from the same galaxy observed with $F W H M$ beams of 1 and $2 \mathrm{kpc}$, respectively, at a low sensitivity with peak $S / N$ of 30 .

However, as the beam-smearing correction is performed by deconvolution - which amplifies the effective noise - it is important to perform beam correction only over the major axis range affected by beam smearing. Otherwise one is adding unnecessary scatter to the measurements across the whole radial extent of the galaxy. To investigate the effect of beam-smearing on the measurements kinematics, simulations we analysed simulated, noise-less XV maps. Figures $5 \mathrm{a}$ and $\mathrm{b}$ show the results of fits when beam-smeared with a telescope beam with an $F W H M_{\theta}$ of 1.0 and $2.0 \mathrm{kpc}$ beams. The beam-smearing effect becomes quite dramatic in the measurements of galaxies observed with large beams ( $2 \mathrm{kpc}$ or more). Because of the location of the grid points, the uncorrected kinematics shows some marked asymmetries (e.g. Fig. 5a) which are almost completely corrected by the beam-smearing correction process (Figs. 6a and 7a).

As explained in Sect. 3.2, beam-smearing causes the velocity profile of a slice to deviate from a sum of Gaussians. As a result, the measured fits of the velocity dispersion and rotation velocity oscillate around the true value, due to the progressive nature of the fitting process. Similar oscillations also occur in the fitted surface density; however these oscillations are on flux scales lower than the scatter due to noise.

The best procedure is to first perform an initial radial decomposition fit of the galaxy without beam-smearing correction. This gives accurate kinematic fits at radii where there is no large intrinsic gradients of the kinematics. The radii affected by beamsmearing are determined from the radii with oscillating measured fits. Subsequent iterations of the fitting process, are performed using beam smearing in this radial range. As explained in Sect. 3.2, the shape of the inner galaxy kinematics are estimated by extrapolating inwards to build the mini-XV diagram used in beam-correcting the radii affected by beam-smearing. Fortuitously, the large radial gradients of the intrinsic kinematics that necessitate beam smearing correction occur predominantly in the inner disk. Most late-type, gas-rich galaxies contain sufficient $\mathrm{HI}$ in the inner disk to apply the beam smearing correction method.

Figures $6 \mathrm{a}$ to $9 \mathrm{a}$ show the measured kinematics fits obtained with beam-smearing correction of XV maps obtained from moderate $\left(F W H M_{\theta}=1.0 \mathrm{kpc}\right)$ and low spatial resolution $\left(F W H M_{\theta}=2.0 \mathrm{kpc}\right) \mathrm{XV}$ maps. The measurements 
J. C. O'Brien et al.: The dark matter halo shape of edge-on disk galaxies. II.
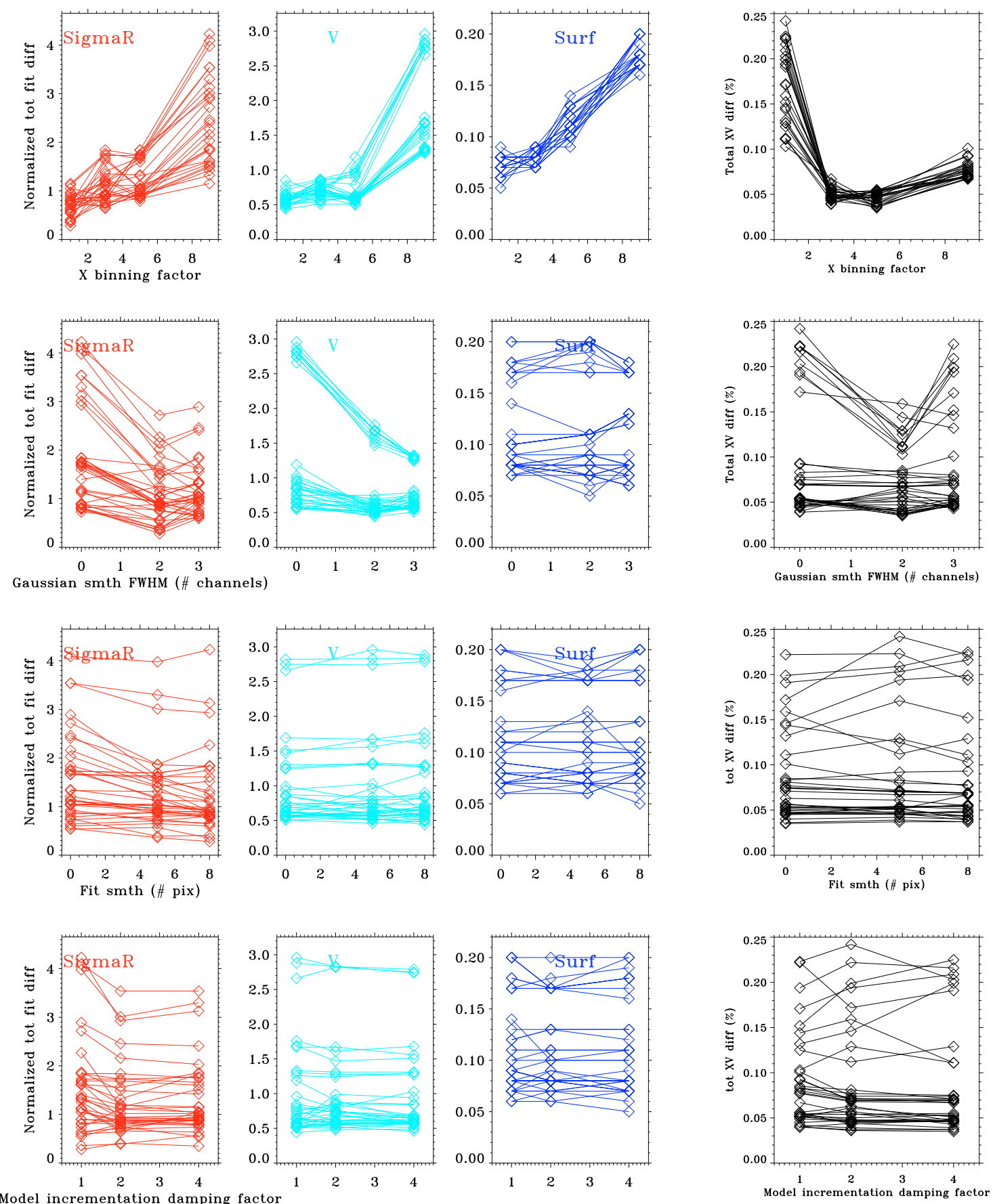

Fig. 10a. These plots display measures of the fit to the kinematics and how these are affected by varying the iterative onion peeler for a synthetic galaxy with signal-to-noise $S / N=80$, and a small beam $\theta_{\text {syn }}=0.3 \mathrm{kpc}$. The first three columns show the integrated absolute difference between the final model and the the original XV diagram for each kinematic function (from left to right respectively velocity dispersion "SigmaR", rotation velocity " $V$ " and surface density "Surf"), normalised by the number of sightlines within a fit. The units for the total fit difference of the velocity dispersion and the rotation curve are in $\mathrm{km} \mathrm{s}^{-1}$, while the surface density difference is measured in log flux. The fourth column displays the integrated flux difference between the final XV diagram built from the best fit kinematics, and the original XV diagram, as a percentage of that in the original XV diagram. See the text for further explanation.

are undertaken on both low and high sensitivity $\mathrm{XV}$ maps (peak $S / N=30$ and $S / N=80$, respectively) at each spatial resolution. The XV maps built with the measured kinematics are displayed in Figs. $6 \mathrm{~b}$ to $9 \mathrm{~b}$.

The residual effects of beam-smearing after correction in high $S / N$ data (peak $S / N=80$ ) can be appreciated by comparing Figs. $4 \mathrm{~b}$ (300 pc beam), 6a (1 kpc beam) and $8 \mathrm{a}(2 \mathrm{kpc}$ beam).
It can be seen that the beam correction process does a good job of correcting the beam-smearing that occurs over the inner disk. The velocity dispersion is well recovered over large radial scales, except for the measurements on the low signal-to-noise $(S / N=30)$ dataset observed with the $1 \mathrm{kpc}$ beam. Very high signal-to-noise observations $(S / N \gtrsim 100)$ were able to recover the radial velocity dispersion structure on sub-beam scales to 

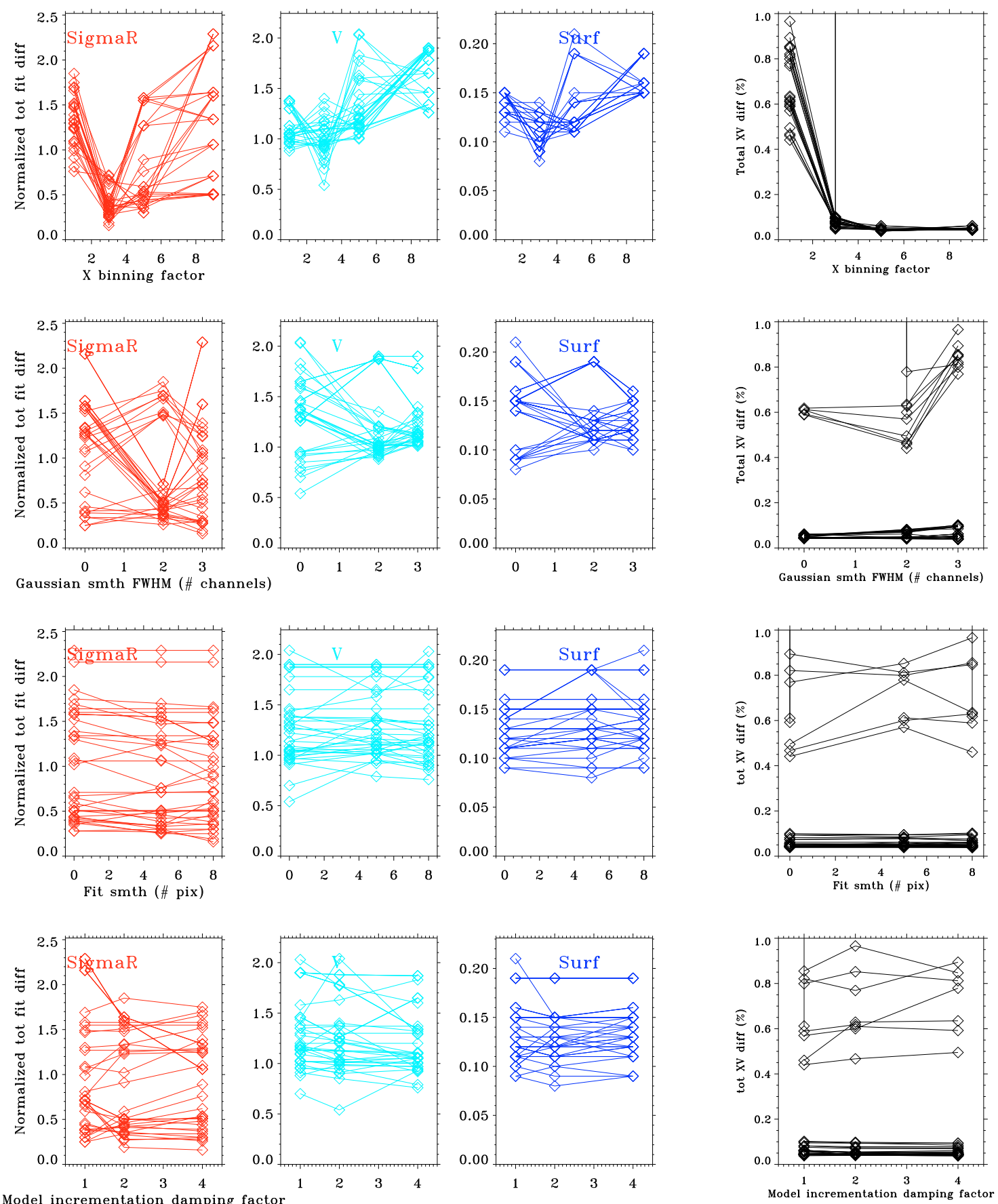

Fig. 10b. These plots display the same measures of the fit to the kinematics as in Fig. 10a, but now for a synthetic galaxy with signal-to-noise $S / N=30$. Lay-out and units are the same as in Fig. 10a.

accuracies of less than $1 \mathrm{~km} \mathrm{~s}^{-1}$, however measurements to observations with large beams $F W H M_{\theta} \geq 1 \mathrm{kpc}$ at $30 \lesssim S / N \lesssim 100$ are limited to recovered the velocity dispersion on radial scales of $\sim 1 F W H M_{\theta}$ and an uncertainty of approximately $1-2 \mathrm{~km} \mathrm{~s}^{-1}$.

The measured rotation curve is accurately recovered. The surface density is also recovered with good accuracy, though the uncertainty is more sensitive to the noise level of the $\mathrm{XV}$ map.

\subsection{Fine tuning the iterative method}

To minimise the noise sensitivity of the velocity dispersion fitting method, various adaptions were trialed on simulated observations with different spatial resolutions and noise levels. These adaptions trialed were:

- Binning of the $R^{\prime}$ dimension of the XV map prior to fitting to increase the signal-to-noise.

- Smoothing of each velocity profile slice through the XV map by a Gaussian of known dispersion prior to fitting kinematics to each velocity slice. Profile broadening by a Gaussian with varying $F W H M$ from 3 to 6 velocity channels was tried. This additional known source of velocity dispersion was taken into account in the derivation of the HI velocity dispersion. 
- Smoothing of the derived fit $\sigma_{v, \mathrm{HI}}(R)$ with a Gaussian $F W H M$ of varying number of $R^{\prime}$ pixels. The smoothed fits were then compared to determine the new model $\sigma_{v, \mathrm{HI}}(R)$. Only the velocity dispersion fit was smoothed, as the rotation curve and deprojected surface density fits exhibited a very low scatter.

- Damping of the model incrementations $\Delta$ (see Fig. 3) to minimise oscillations in the fits used to create the new model. Damping was only applied to the model incrementation of the velocity dispersion, as the small scatter of the fits to the rotation curve and HI surface density removed the need for any model damping.

Several variations were tried for each adaption, resulting in 108 separate simulations, which were run on both a high $(S / N=80)$ and low $(S / N=30)$ signal-to-noise XV map observed with a $0.3 \mathrm{kpc}$ beam. The galaxy model used was a exponential HI surface density, constant HI velocity dispersion and a differential rotation curve. Two indicators of the accuracy were measured.

Firstly, a residual XV diagram was calculated by subtracting the original XV diagram from the XV diagram formed from the best fit kinematics. The summed absolute residual XV flux in $\%$ of the total flux in the maps is displayed in the plots in the far right column of Fig. 10a for the $S / N=80$ results, and Fig. $10 \mathrm{~b}$ for the $S / N=30$ results. While the residual $\mathrm{XV}$ diagram is a good indicator of measurement accuracy on large scales, it is quite insensitive to measurement errors on small radial scales. Except for one fit which diverged in the simulations on low signal-to-noise XV diagram, the summed residual was not more than $1.0 \%(0.25 \%$ in the SN80 dataset) of the total flux in the original map for both the low and high $S / N$ measurements, being approximately three times greater in the fit to the low signal-to-noise map.

Secondly, we also used the difference between the kinematics measured from the XV diagram formed with the best fit kinematics and the kinematics measured from the observed XV diagram. The difference in fit (again absolute values) at each radius was then summed to form a total difference for the fit to that kinematic function. We were able to compare the fits obtained from binned and un-binned XV maps, by normalising the total difference in fit by the number of sightlines used in the fit. The normalised total fit difference for each kinematic function is displayed in the plots in the first three columns of Fig. 10a for the $S / N=80$ results, and Fig. 10 b for the $S / N=30$ results.

Each line marks the trend in the normalised total fit difference if only the method plotted on the abscissa is varied, and all other method variations are held constant. Sometimes the diamond symbols overlap, giving the impression that there are fewer simulations in some of the panels. However, the number of lines in each panel is the same and the variation of parameters is distributed evenly over the various panels. The large spread in normalised total fit difference of the different lines is primarily due to binning the XV diagram along the major axis prior to fitting. In the plots showing how the measurements varied with other method variations, the lines with high measurement error are those which used a large major axis binning interval. As expected, binning the XV diagram prior to fitting reduced the amount of major axis structure information, which dramatically increased the measurement uncertainty of all three fitted functions. The different values of the binning interval have in some cases resulted in the outcomes being distributeded in two somewhat distinct groups.

The plots show that smoothing the fits before incrementing, and damping the model incrementation offer no improvement.
For the high signal-to-noise dataset, the velocity dispersion measurements were improved by smoothing the velocity profile of each slice by a small Gaussian prior to fitting. The velocity dispersion measurements to the peak $S / N=80$ dataset were improved by $25 \%$ (or on average $0.5-1.0 \mathrm{~km} \mathrm{~s}^{-1}$ ) with a Gaussian smoothing kernel with a $F W H M$ of 2 velocity channels, i.e. slightly larger than the $F W H M$ of noise which will typically be 1-2 channels. However, Gaussian smoothing the velocity axis caused no distinct improvement in the measurements to the low signal-to-noise dataset. This is probably because Gaussian smoothing a noisy profile distorts the shape slightly by incorporating the noise into the smoothed profile shape. Consequently, we have not adopted any additional smoothing or other variations discussed in this section (Sect. 4.4) to the radial decomposition method.

\subsection{Accuracy of the iterative radial decomposition method}

Convergence to the best kinematic model was usually achieved after three iterations of the radial decomposition fitting process. The average measurement errors of each kinematic fit determined with the adopted unchanged radial decomposition method are: $\Delta \sigma_{\mathrm{v}, \mathrm{HI}}=0.7,1.3 \mathrm{~km} \mathrm{~s}^{-1}$ (for peak $S / N=80$ and peak $S / N=$ 30 , respectively); $\Delta V=0.9,1.4 \mathrm{~km} \mathrm{~s}^{-1}$, and $\Delta\left(\log \Sigma_{\mathrm{HI}}\right)=0.1$, 0.15 . The sum of the residual XV diagram shows that the radial decomposition level was able to recover the flux to a level of $0.2 \%$ and $0.6 \%$ of the total flux of the observed XV diagram (for maps with peak $S / N=80$ and $S / N=30$, respectively). This measurement accuracy is indicative only, as it constitutes a mean error to measurements of a galaxy with this particular kinematics and structure. As mentioned earlier, fits to a XV map observed to the same sensitivity level of a galaxy with a less steep intrinsic surface density should have a lower measurement error.

\section{Summary}

Using our new iterative radial decomposition method, we show that it is possible to accurately measure all three functions that describe the planar kinematics and distribution of a galaxy: the radial HI surface density, the rotation curve and the HI velocity dispersion. This method is a significant improvement on previous methods to model edge-on galaxies which approximated one or more of these kinematic functions. Measurements of simulated galaxies show that a peak signal-to-noise of $\gtrsim 30$ is needed to accurately measure all three kinematic functions on a galaxy with a intrinsically steep (exponential over all $R$ ) HI surface density. However, reliable measurements would probably also be obtained with observations at a peak signal-to-noise $(S / N \gtrsim 20)$ Furthermore, the method should also be successful on galaxies with an intrinsically shallower surface density - as is common in most disk galaxies due to depletion of $\mathrm{HI}$ in the stellar disk.

In the next paper in this series, we present the derived Hi kinematics and radial surface density of our galaxies as measured with this method. Paper III also contains the vertical HI flaring results, which is measured as a function of galactocentric radius using the deprojected HI distribution and kinematics.

Acknowledgements. We are very grateful to Albert Bosma who contributed greatly to initiating this project. He pointed out that HI flaring studies are best done on edge-on galaxies with low maximum rotational velocities, and we used an unpublished Parkes HI survey of edge-on galaxies by Bosma and $\mathrm{KCF}$ when selecting our galaxies. J.C.O. thanks E. Athanassoula, M. Bureau, R. Olling, A. Petric and J. van Gorkom for helpful discussions. J.C.O. is grateful to 
B. Koribalski, R. Sault, L. Staveley-Smith and R. Wark for help and advice with data reduction and analysis. We thank the referee, J. M. van der Hulst, for his careful and thorough reading of the manuscripts of this series of papers and his helpful and constructive remarks and suggestions.

\section{References}

Binney, J., \& Tremaine, S. 1987, Galactic Dynamics (Princeton University Press) Bottema, R., Shostak, G. S., \& van der Kruit, P. C. 1986, A\&A, 167, 34

Boulanger, F., \& Viallefond, F. 1992, A\&A, 266, 37

de Blok, W. J. G., \& Bosma, A. 2002, A\&A, 385, 816

Dickey, J. M., Hanson, M. M., \& Heloe, G. 1990, ApJ, 352, 522

Fall, S. M., \& Efstathiou, G. 1980, MNRAS, 193, 189

Fraternali, F., van Moorsel, G., Sancisi, R., \& Oosterloo, T. 2002, AJ, 123, 3124 Fraternali, F., Oosterloo, T. A., Sancisi, R., \& Swaters, R. 2005, ASP Conf., 331, 239

García-Ruiz, I., Sancisi, R., \& Kuijken, K. H. 2002, A\&A, 394, 769

Kregel, M., \& van der Kruit, P. C. 2004, MNRAS, 352, 787

Kregel, M., van der Kruit, P. C., \& de Blok, W. J. G. 2004, MNRAS, 352, 768

Lo, K. Y., Sargent, W. L. W., \& Young, K. 1993, AJ, 106, 507
Mathewson, D. S., Ford, V. L., \& Buchhorn, M. 1992, ApJS, 81, 413

Nelder, J. A., \& Mead, R. 1965, Computer J., 7, 308

O’Brien, J. C., Freeman, K. C., van der Kruit, P. C., \& Bosma, A. 2010, A\&A, 515, A60 (Paper I)

Olling, R. P. 1996, AJ, 112, 457

Petric, A. O., \& Rupen, M. P. 2007, AJ, 134, 1952

Press, W. H., Teukolsky, S. A., Vetterling, W. T., \& Flannery, B. P. 1992, Numerical Recipes in FORTRAN - The Art of Scientific Computing (Cambridge University Press)

Rownd, B. K., Dickey, J. M., \& Helou, G. 1994, AJ, 108, 1638

Rubin, V. C., Burstein, D., Ford, W. K., \& Thonnard, N. 1985, ApJ, 289, 81

Sancisi, R., \& Allen, R. J. 1979, A\&A, 74, 73

Shane, W. W., \& Bieger-Smith, G. P. 1966, Bull. Astron. Inst. Neth., 18, 263

Shostak, G. S., \& van der Kruit, P. C. 1984, A\&A, 132, 20

Sofue, Y. 1986, ApJ, 458, 120

Sofue, Y., Koda, J., Nakamishi, H., \& Onodera, S. 2003, PASJ, 55, 59

Swaters, R. S. 1999, Ph.D. Thesis, Groningen

Swaters, R. S., Sancisi, R., \& van der Hulst, J. M. 1997, ApJ, 491, 140

Takamiya, T., \& Sofue, Y. 2002, ApJ, 576, L15

Uson, J. M., \& Matthews, L. D. 2003, AJ, 125, 2455

van der Kruit, P. C. 1981, A\&A, 99, 298

van der Kruit, P. C., \& Shostak, G. S. 1982, A\&A, 105, 351

van der Kruit, P. C., \& Shostak, G. S. 1984, A\&A, 134, 258

Warmels, R. H. 1988, A\&AS, 72, 427 